\title{
Effect of Axial Diffusion on the Response of Diffusion Flames to Axial Flow Perturbations*
}

\author{
Nicholas A. Magina ${ }^{\mathrm{a}}$ and Timothy C. Lieuwen ${ }^{\mathrm{b}}$ \\ ${ }^{\mathrm{a}, \mathrm{b}}$ School of Aerospace Engineering \\ Georgia Institute of Technology \\ Atlanta, GA, USA 30332 \\ Corresponding author: Nicholas A. Magina \\ Corresponding author email address: narthurm@gatech.edu \\ Phone: 443-848-1404 \\ Fax: 404-385-7117 \\ Ben T. Zinn Aerospace Combustion Laboratory \\ School of Aerospace Engineering \\ Georgia Institute of Technology \\ 635 Strong St NW, Atlanta, GA, 30318 \\ Full-length article / Accepted Paper \\ Black-and-white printing
}

${ }^{*}$ Expanded from AIAA conference paper (DOI number 10.2514/6.2014-0651) presented at SciTech 2014 at the National Harbor, Maryland.

${ }^{a}$ Graduate Research Assistant, GT AE Comb Lab, 635 Strong St, ATL, GA, 30318

${ }^{\mathrm{b}}$ Professor, GT AE Comb Lab, 635 Strong St, ATL, GA, 30318

(C) 2016. This manuscript version is made available under the Elsevier user license

http://www.elsevier.com/open-access/userlicense/1.0/ 


\begin{abstract}
This paper elucidates the behavior and dynamics of non-premixed flames responding to bulk fluctuations in flow velocity. It expands previous work on this problem by consistently incorporating finite Peclet number ( $P e=U_{0} R_{f} / \mathscr{D}$ ) effects, and differentiating inflow boundary and dynamical effects on the flame dynamics. For analytical tractability, prior treatments of this problem generally prescribe the inflow boundary conditions into the domain. This paper shows, however, that prescribed inflow conditions, such as a step or constant local diffusive flux boundary condition, neglect axial diffusion effects in the region where their effects are most important; i.e., in the near-burner exit region where high transverse gradients and mass burning rates control the heat release dynamics. As the burning rate of non-premixed flames are controlled by mixture fraction gradients, the influence of axial diffusion substantively influences several burning rate characteristics of the flame. In addition, these effects cause the leading edge position of the flame front to oscillate, even for infinitely fast chemistry. Even in $P e>>1$ flames, axial diffusion introduces several fundamentally new features to the problem, resulting in exponentially decaying, dispersive flame wrinkle propagation with downstream distance. Also investigated here are asymptotic results for the heat release dynamics. It is shown that axial diffusion introduces a triple-zone asymptotic structure into the unsteady heat release characteristics, resulting in $O(1)$ flame transfer function trends for $S t<<1$ (to which an $n-\tau$ model is developed), $O\left(1 / S t^{1 / 2}\right)$ for intermediate Strouhal numbers, and $O(1 / S t)$ for high Strouhal numbers. Finally, it is shown that the phase of the heat release response is approximately half that of a premixed flame with the same length, due to the concentration of unsteady heat release near the burner outlet, where transverse gradients are largest.
\end{abstract}

Keywords

Non-premixed flame, linear flame response, velocity coupled response, combustion instabilities, flame transfer function, axial diffusion effects

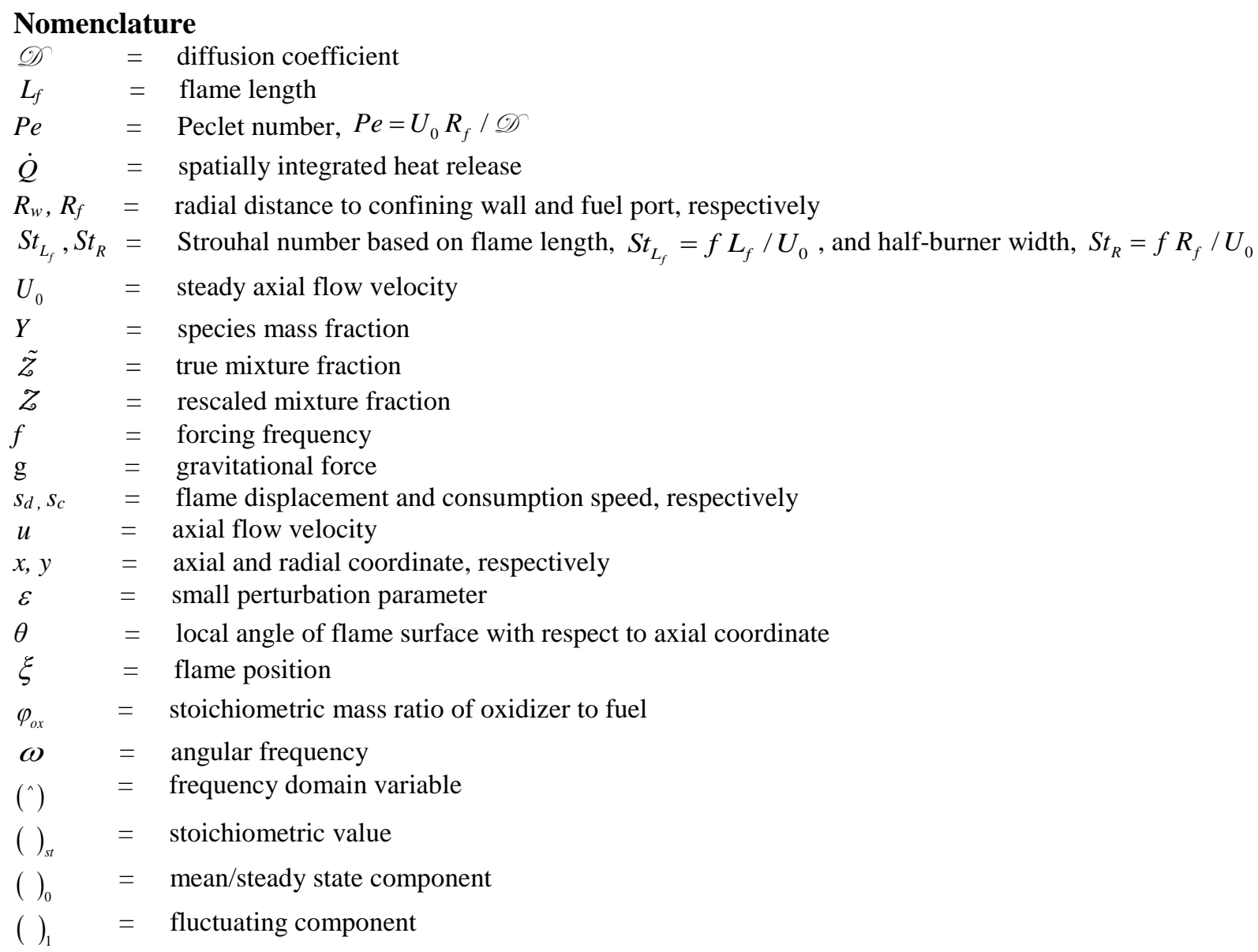




\section{Introduction}

This paper investigates the features and dynamics of non-premixed flames responding to spatially uniform fluctuations in flow velocity. It is motivated by performance and safety concerns arising from combustion instabilities, which manifest as large amplitude heat release oscillations associated with one or more combustor acoustic modes [1]. This study concentrates on the velocity-coupled part of the feedback loop, analyzing the flames response to flow disturbances. The response of premixed flames to flow disturbances and the combustion instability characteristics of lean, premixed combustors has been extensively investigated and documented over the last few decades [1-8]. In contrast, the behavior of non-premixed flames responding to flow disturbances, both in regards to the space-time reaction sheet and temporally varying heat release dynamics, both local and spatially integrated, has received significantly less attention. Non-premixed, or partially premixed flames, however, are routinely encountered in liquid-fueled combustion systems, as well as in pilot systems for premixed combustors, and industrial operators routinely note the profound influence of non-premixed pilot fuel on combustor oscillation limits.

A number of studies have delved into the natural dynamics of non-premixed flames. In the buoyancy dominated regime where the Froude number, $F r=U_{0}^{2} /\left(\mathrm{g} R_{f}\right)$, is not too high, these flames are globally unstable and exhibit narrowband oscillations due to the periodic generation and traveling of vortical structures vertically along the flame. These are manifested as flame flicker at a low frequency $(\sim 12 \mathrm{~Hz})$ that is remarkably insensitive to flow rate, burner size, and gas composition $[9,10]$. The amplitude of spatial flickering is, however, a function of these parameters. Recent studies have shown that this global instability disappears at small Froude numbers, or when the flame becomes momentum dominated at large Froude numbers [11].

External excitation of non-premixed flames, such as by acoustic forcing, has also been studied extensively, often with the motivation of enhancing mixing and/or decreasing pollutant emissions. When subjected to external excitation, lower Froude number, nominally unstable flames exhibit a variety of response features that depend upon the frequency and magnitude of the excitation. For example, Chen et al. studied the response of a non-premixed flame exposed to acoustic excitation [12], showing oscillations in both the fuel jet flow and flame sheet position, both of which were dependent upon the forcing frequency and amplitude. They and others [13-15] also showed nonlinear behavior, such as the presence of sum and difference frequencies of the buoyant instability and external forcing frequencies, subharmonics and harmonics of the excitation frequency, and frequency locking - i.e., the disappearance of oscillations associated with the natural buoyant instability at sufficient excitation amplitudes. For example, Williams et al. [16] explored this lock-in behavior, showing that forcing the fuel stream at a frequency close to the natural buoyant instability frequency was accompanied by the presence of large vortices on the air side of the flame, coupling the overall flame response to the forcing frequency. They also observed that a related lock-in phenomenon could happen at the first subharmonic of the forcing frequency, when the forcing frequency was close to twice the natural instability frequency.

As a result of the strong effect of forcing on the ambient/co-flowing air and its entrainment with the fuel jet, a number of studies have also noted significant influences on soot and $\mathrm{NO}_{\mathrm{x}}$ production from the flame [17-19] sensitivities which are much stronger in non-premixed flames than in premixed flames. For example, Saito et al. [17] showed that soot can be suppressed in acoustically excited non-premixed flames, with reductions of up to 50\% in a laminar flame, and $90 \%$ for a turbulent flame.

Additional studies have looked into the dynamics of laminar, momentum dominated flames, focusing on the flame's space-time dynamics due to velocity and equivalence ratio perturbations. Forced flow disturbances produce a spatially varying, oscillatory velocity field whose component that is normal to the flame causes wrinkling and pocket formation beyond critical conditions, as well as oscillatory reaction and heat release rates [19-21].

A number of analytical studies have also considered the response of momentum dominated non-premixed flames. A significant theoretical literature on the unforced problem exists, the Burke-Schumann flame being a classic problem, along with works distinguishing the effects of streamwise diffusion [22, 23]. Several treatments of the forced, unsteady problem have been reported, in particular those of Sujith [24-26], Chakravarthy [21, 26], Juniper et al. [27], and Magina et al. [28, 29]. These studies have analyzed this problem within the infinite reaction rate, $z$-equation formulation for the mixture fraction. Solutions were developed for the flame position and heat release for several problems, including the flame response to axial velocity and mixture fraction oscillations.

This study closely follows the work of Magina et al. [28, 30, 31] which primarily focused on large Peclet number flames, i.e. where axial transport is dominated by convection relative to diffusion, a limit which significantly simplifies the analysis and enables the development of explicit solutions for the space-time dynamics of the flame position and unsteady heat release. It was shown that the oscillatory flow velocity component normal to 
the flame was responsible for exciting wrinkles on the flame sheet, whose magnitude had strong axial dependence and monotonically decreasing phase. In the absence of axial diffusion, these wrinkles convect axially at the mean flow speed, $U_{0}$, with constant amplitude and phase speed. Axial diffusion effects cause these wrinkles to dissipate as they convect downstream, reducing the overall wrinkle amplitude and resulting in imperfect interference. Finally, they showed that the spatially integrated unsteady heat release was dominated by mass burning rate fluctuations, rather than area fluctuations, and rolled off much slower with frequency, $\mathrm{O}\left(S t^{-1 / 2}\right)$ compared to $\mathrm{O}\left(S t^{-1}\right)$ for premixed flames, indicating non-premixed flames are much more sensitive to flow perturbations at intermediate Strouhal numbers.

Several questions still remain about the flame position and heat release dynamics of finite Peclet number flames. In particular, axial diffusion effects manifest themselves in a variety of ways, not all of which have been captured in prior analyses. Most theoretical analyses of the problem impose inflow conditions on the mean and fluctuating solutions, even in studies that capture axial diffusion effects within the domain itself. For example, our earlier study that demonstrated how axial diffusion introduced damping of flame wrinkles utilized a step-inlet boundary condition [29]. This simplification introduces a singularity in the solution, as there is an infinite gradient in mixture fraction at the fuel port lip. As we will show here, the high frequency characteristics of the heat release are quite sensitive to the inflow profile, and the step-inflow boundary condition leads to incorrect conclusions on these asymptotic characteristics of the heat release transfer function, even in the $P e>>1$ limit. Stated differently, specifying an inflow step boundary conditions neglects axial diffusion effects in the region where these effects are most important - in the near-burner exit region where high transverse gradients and mass burning rates control the heat release dynamics. Thus, a key contribution of the present study is to consistently capture finite $P e$ effects. As will be shown, this requires computational solutions of the governing equations, as explicit analytical solutions are not possible in this case.

A recent paper by Xiong et al. [32] on buoyancy dominated flames has also emphasized the significance of the inflow conditions on coflow diffusion flame solutions. Similar to this study, they specified the fuel inlet boundary condition well upstream of the inflow, and showed how axial diffusion effects influenced the flame position. In addition, they showed how buoyancy forces exerted on the fuel and burnt gas significantly distorted the near-nozzle flow-fields.

The rest of this paper is organized as follows; first we introduce the governing equations and key assumptions for the mixture fraction fields along with the appropriate geometry for studying the complete and consistent inclusion of axial diffusion effects. Next we discuss how axial diffusion effects manifest themselves in the mixture fraction field and flame dynamics. Its influence on the inflow and inlet conditions as well as on the flame attachment point will be studied. Then we observe the modifications in space time dynamics and heat release analysis that axial diffusion introduces. By comparing these results to our previous $P e \rightarrow \infty$ studies, we can isolate the various effects that axial diffusion has on the flame dynamics. Comparisons with similar configuration premixed flames will also be made for various dynamically significant quantities in order to examine controlling physics between these two flame types.

\section{Formulation}

This section presents the basic formulation of the problem, summarizing several key observations from our earlier works for completeness [28, 29,33]. As in these earlier studies, the key assumptions of this analysis are (1) infinitely fast chemistry, wherein the flame sheet collapses to the $Z_{s t}$ surface, (2) all species have equal diffusivities, (3) negligible radiative heat loss effects, (4) constant diffusion coefficients, and (5) small perturbation amplitudes. In addition, we impose the steady and fluctuating velocity fields, decoupling the momentum equation from the energy and species equations and, thus, implicitly assume an isothermal field. Following assumption (2), the space-time dynamics of a non-premixed flame with constant species diffusivities can be described by the mixture fraction field utilizing the $z$-equation, as shown by Eq.(1) [34]:

$$
\frac{\partial \tilde{z}}{\partial t}+\vec{u} \cdot \nabla \tilde{Z}=\nabla \cdot(Q \nabla \nabla \tilde{Z})
$$

Employing assumption (1), the reaction sheet becomes coincident with the $Z_{s t}$ surface, defined by the expression $\tilde{z}(\vec{x}, t)=\tilde{z}_{s t}=1 /\left(1+\varphi_{o x}\right)$, where $\varphi_{o x}$ denotes the stoichiometric mass ratio of oxidizer to fuel. Although the 
field solutions are significant, it is the dynamics of this reaction sheet, both instantaneous position (given by $y=\xi\left(x, \tilde{z}_{\mathrm{st}}, t\right)$ in Figure 1) and corresponding heat release that we are specifically interested in. This assumption will also be imposed implicitly in Section 3, as the reaction sheet is defined and persists all the way back to the burner. In reality, finite-rate effects due to high local mixture fraction gradients could lead to local-extinction and flame lift-off in the near burner region where the scalar dissipation rate is significantly large.

Recognizing the additional challenges related to obtaining explicit expressions for non-premixed flame reaction sheet dynamics, compared to premixed flame expressions [28], following assumption (5) we examine linearized solutions to the $Z$-equation, valid in the limit of small perturbation amplitudes. Thus, each variable can be expanding into the following form:

$$
()(x, t)=()_{0}(x, y)+()_{1}(x, y, t)
$$

Neglecting higher order terms, the steady state, i.e. unforced, mixture fraction field, for a system with no transverse flow, can be acquired from Eq.(1) as:

$$
U_{0} \frac{\partial{\tilde{z_{0}}}_{0}}{\partial x}=\mathscr{O} \frac{\partial^{2}{\tilde{Z_{0}}}_{0}}{\partial y^{2}}+b \mathscr{O} \frac{\partial^{2} \tilde{\tilde{Z}_{0}}}{\partial x^{2}}
$$

where " $b$ " is an axial diffusion indicator, taking values of unity and nil, depending upon whether axial diffusion effects are enabled or disabled, respectively. Likewise, the dynamical equation for the fluctuating, i.e. forced, mixture fraction field can be written in the frequency domain as:

$$
-i \omega \hat{\tilde{z_{1}}}+U_{0} \frac{\partial \hat{\tilde{z_{1}}}}{\partial x}-\mathscr{O} \frac{\partial^{2} \hat{\tilde{z_{1}}}}{\partial y^{2}}-b \mathscr{O} \frac{\partial^{2} \hat{\tilde{z}}_{u_{1}}}{\partial x^{2}}=-\hat{u}_{x, 1} \frac{\partial \tilde{z}_{0}}{\partial x}-\hat{u}_{y, 1} \frac{\partial \tilde{z}_{0}}{\partial y}
$$

As is shown in Figure 1, the domain of interest is accompanied by two reservoirs, one consisting of pure fuel and one pure oxidizer, both located at $x=-\infty$, where:

$$
\tilde{Z}(x=-\infty, y)= \begin{cases}\tilde{Z}_{f, r e s} & 0<|y|<R_{f} \\ \tilde{Z}_{o x, \text { res }} & R_{f}<|y|<R_{w}\end{cases}
$$

Previous theoretical analysis of the unsteady problem have specified the inflow conditions of fuel and oxidizer at $x=0$. However, to properly include and account for axial diffusion effects, and to properly describe the fuel/oxidizer gradients near the burner lip, we must include the $x<0$ fuel and oxidizer ports, which provide solution domains for back diffusion to occur, altering the inlet profile. Thus, these field equations are solved employing the extended inlet geometry, shown in Figure 1, subjected to the above boundary condition. In addition, no-penetration side/fuel port wall boundary conditions are applied and finite mixture fraction values are required at all physical axial locations. For clarity, we define a rescaled mixture fraction variable as:

$$
z=\frac{\tilde{z}-\tilde{Z}_{o x, r e s}}{\tilde{Z}_{f, r e s}-\tilde{Z}_{o x, r e s}}
$$

This paper considers the axially forced configuration (i.e. $u_{y, 1}=0$ in Eq.(4)). The problem is considered both analytically, where explicit solutions are possible in cases where the inflow boundary condition at $x=0$ is specified, and computationally, for the general problem where the inflow conditions at $x=0$ must be solved simultaneously with the rest of the domain of interest. As we will show in the next section, explicitly specifying an inflow step boundary condition neglects axial diffusion effects in the region where they are most important, and introduces a non-physical infinite transverse gradient in mixture fraction at the fuel port lip. Likewise, specifying a local diffusive flux boundary condition imposes a discontinuity in local mass flux between the fuel/oxidizer ports and combustion solution domains; both of whose effects will be discussed later. 
The computational solutions were obtained with finite element methods, using the Comsol Multiphysics solver due to its balance of computational freedom and efficiency. The multifrontal massively parallel sparse direct solver, denoted MUMPS, was utilized with a convergence criterion set to $10^{-5}$ and relative tolerance of 0.001 . This direct solver is based on LU decomposition and can take advantage of all processor cores for increased computational speed. Also, since our previous studies revealed large spatial gradients in the near burner lip region, a mapped rectangular mesh was employed, containing increased grid resolution closer to the fuel port lip. The maximum element size, occurring near the exit plane was on the order of $R_{f}$, while the minimum element size, occurring near the fuel port lip, was on the order of $R_{f} / 10^{3}$. Dirichlet and Neumann boundary conditions were utilized at the inlet/outlet and at the port/walls, respectively. Solution results were fortified by grid sensitivity studies, which showed that an increase in mesh density by 9 , yielded less than $2 \%$ modification in field and contour results.

\section{Results and Discussion}

This section is organized into four sub-sections. Section 3.1 first considers inflow conditions. Previous investigations into non-premixed flame dynamics were performed in the large $\mathrm{Pe}$ limit, although this assumption was not always explicitly stated. For example, most studies utilized a step inlet boundary condition for inflow mixture fraction at $x=0$. However, when considering finite/lower $P e$ value effects and, thus, axial diffusion effects, this inlet boundary condition neglects the time varying back diffusion of products and alternate reactant into each respective fuel/oxidizer port. Section 3.2 then considers the location of the flame at the burner and discusses the oscillatory attachment point induced by axial diffusion effects. Then, Section 3.3 considers the space-time dynamics of the reaction sheet and Section 3.4 considers the spatially integrated heat release.

\subsection{Inflow and Inlet Conditions}

This section further discusses the inflow conditions and their relation to the inlet (defined as the $x=0$ plane) conditions, denoted as $Z_{0}(x=0, y)={ }_{e} f_{0}(y)$ and $Z_{1}(x=0, y)=f_{1}(y)$. Standard solution techniques can be used to solve Eqs.(3) and Eq.(4) for a given steady and fluctuating inflow profile, $f_{0}(y)$ and $f_{1}(y)$, respectively for spatially uniform forcing. Defining our forcing configuration as spatially uniform axial velocity fluctuations, represented by $u_{x, 1}=\varepsilon U_{0} \exp [-i \omega t]$, and including axial diffusion in the governing equations by setting $b=1$, mixture fraction field solutions for $x>0$ can be obtained:

$$
\begin{gathered}
\mathcal{Z}_{0}=A_{0}+\sum_{n=1}^{\infty} A_{n} \cos \left(\mathscr{A}_{n} \frac{y}{R_{f}}\right) \exp \left(\mathscr{B}_{-} \frac{x}{P e R_{f}}\right) \\
\hat{\mathcal{Z}}_{1}=\sum \frac{\varepsilon A_{n} \mathscr{B}_{-}}{2 \pi i P e S t_{R}} \cos \left(\mathcal{A}_{n} \frac{y}{R_{f}}\right) \exp \left[\mathscr{B}_{-} \frac{x}{P e R_{f}}\right]+\sum B_{n} \cos \left(\mathscr{A}_{n} \frac{y}{R_{f}}\right) \exp \left[\mathscr{B}_{h} \frac{x}{P e R_{f}}\right]
\end{gathered}
$$

where $\mathcal{A}_{n}=n \pi\left(R_{f} / R_{w}\right)$ are eigenvalues and $A_{0}, A_{n}$, and, $B_{n}$ are given by:

$$
\begin{gathered}
A_{0}=\frac{1}{R_{w}} \int_{0}^{R_{w}} f_{0}(y) d y \\
A_{n}=\frac{2}{R_{w}} \int_{0}^{R_{w}} f_{0}(y) \cos \left(\mathcal{A}_{n} \frac{y}{R_{f}}\right) d y \quad B_{n}=\frac{2}{R_{w}} \int_{0}^{R_{w}} f_{1}(y) \cos \left(\mathcal{A} \frac{y}{R_{f}}\right) d y-\frac{\varepsilon A_{n} \mathscr{B}_{-}}{2 \pi i P e S t_{R}}
\end{gathered}
$$

and where $\mathscr{B}_{-}$and $\mathscr{B}_{h}$ are defined as follows:

$$
\mathscr{B}_{-}=\frac{P e^{2}-\sqrt{P e^{4}+4 P e^{2} \mathscr{A}_{h}^{2}}}{2}
$$




$$
\mathscr{B}_{h}=\frac{P e^{2}-\sqrt{P e^{4}+4 P e^{2} A_{n}^{2}-8 \pi i P e^{3} S t_{R}}}{2}
$$

The $b=0$ solutions can be obtained by taking the $P e \rightarrow \infty$ limit of these solutions. As noted above, most previous studies have utilized a step inflow condition [28] for mathematical ease and tractability, represented as:

$$
f_{0}(y)= \begin{cases}1 & 0 \leq|y|<R_{f} \\ 0 & R_{f} \leq|y|<R_{w}\end{cases}
$$

and

$$
f_{1}(y)=\{0 \quad \forall y
$$

In this limit, the general steady state and fluctuating mixture fraction field solutions, given by Eqns.(7) and (8), can be simplified substantially and result in solutions previously treated in Magina et al. [29]:

$$
\begin{gathered}
Z_{0}=\frac{R_{f}}{R_{w}}+\sum_{n=1}^{\infty} \frac{2}{n \pi} \sin \left(\mathscr{C A}_{n}\right) \cos \left(\mathscr{A}_{n} \frac{y}{R_{f}}\right) \exp \left(\mathscr{B}_{-} \frac{x}{P e R_{f}}\right) \\
Z_{1}=\sum_{n=1}^{\infty}\left[\frac{\varepsilon \sin \left(\mathscr{A}_{n}\right) \mathscr{B}_{-}}{n \pi^{2} i S t_{R} P e}\right] \cos \left(\mathscr{A}_{n} \frac{y}{R_{f}}\right) \exp \left(\mathscr{B}_{-} \frac{x}{P e R_{f}}\right)\left[1-\exp \left(\left\{\mathscr{B}_{h}-\mathscr{B}_{-}\right\} \frac{x}{P e R_{f}}\right)\right] \exp [-i \omega t]
\end{gathered}
$$

While solutions of this form are useful for analyzing certain dynamical features of the problem, the inflow profiles $f_{0}(y)$ and $f_{1}(y)$ cannot be imposed on the problem in general; rather they must be solved as part of the coupled-domain convection-diffusion problem, and extracted from the computation. This issue is analyzed further in Appendix A.

Figure 2 provides the results of such a computational procedure, for various $P e$ values. Significantly, for large $P e$ values, the inlet mixture fraction profile steepens and sharpens, tending towards the step profile, i.e. Eq.(13), in the $P e>>1$ limit. However, $f_{0}(y)$ is significantly smoothed in the transverse direction with moderate/low values, an effect which grows with decreasing $P e$.

\subsection{Flame "Attachment" Point}

The instantaneous flame attachment point behavior, i.e. the axial location where the reaction sheet intersects the fuel port wall, is also influenced by axial diffusion. This location stays fixed in the $P e \rightarrow \infty$ limit at the fuel port lip ( $x$ $=0$ and $\left.y=R_{f}\right)$ for all $P e$ and $Z_{s t}$ combinations, a result of the imposed inlet boundary condition, $Z_{1}(x=0, y, t)=0$.

However, axial diffusion effects shift the flame attachment point away from the outlet-lip and back into the fuel or oxidizer ports (depending on $Z_{s t}$ and $P e$ values). A detail of the unforced flame position near the burner exit is shown in Figure 3 for various $P e$ values. It can be observed that increasing $P e$ acts to drive all the various isocontour attachment points towards the fuel port lip (all converging to a single point in the $P e \rightarrow \infty$ limit). As $P e$ is decreased these positions move upstream, either into the fuel or oxidizer port, depending upon $Z_{s t}$.

Figure 4 shows a summary plot of the steady state attachment point versus $Z_{s t}$ for various $P e$ values. Positive values represent attachment locations inside the oxidizer port on the top side of the fuel port lip, whereas negative values represent attachment locations inside the fuel port on the bottom side of the fuel port lip. The large $P e$ values show near independence of attachment point on $Z_{s t}$, while the smaller values show large sensitivity in attachment point location to $Z_{s t}$.

The instantaneous attachment point also oscillates axially about its steady location with a magnitude that is dependent upon forcing amplitude and the forcing frequency. The frequency sensitivity is due to the low-pass filter characteristic of the flame position, as the time derivative of the flame position is related to the local flow velocity through Eq.(1). Figure 5 shows this more clearly through magnitude and phase plots of the instantaneous flame 
attachment point transfer function for various dimensionless frequency values, where $\hat{x}_{\text {attach }}$ denotes the complex fluctuating axial position of the flame attachment point. The transfer function, defined as $\left(\hat{x}_{\text {attach }} / R_{f}\right) /\left(\hat{u}_{1} / U_{0}\right)$ quantifies the input-output relation between forcing and flame attachment point motion. In addition to the low pass filter characteristic, the increased motion at low $P e$ values, as also shown by the previous figures, can also be seen. The $Z_{s t}$ value also has an influence upon the transfer functions, an effect which is coupled to $P e$, whose degree can be deduced from Figure 4 . For example, very low and very high $Z_{s t}$ values have larger transfer function amplitudes than intermediate values.

\subsection{Space-Time Dynamics of the Reaction Sheet}

While the solutions for the steady and fluctuating mixture fraction fields, $Z_{0}$ and $Z_{1}$, are of some use, the flame position, coinciding with the locus of points in which $Z(x, \xi(x, t))=Z_{s t}$, as well as the mixture fraction gradients at these locations, are of most interest. Unfortunately, an explicit expression for the flame position, $\xi$, is not generally available due to the implicit form of the mixture fraction solutions. However, an explicit expression for the fluctuating flame position can be obtained via:

$$
\xi_{1, n}(x, t)=-\frac{Z_{1}\left(x, y=\xi_{0}(x), t\right)}{\frac{\partial Z_{0}}{\partial n}\left(x, y=\xi_{0}(x)\right)}=-\frac{Z_{1}\left(x, y=\xi_{0}(x), t\right)}{\left|\nabla Z_{0}\right|_{\left(x, y=\xi_{0}(x)\right)}}
$$

where $\xi_{1, n}$ is measured normally, into the oxidizer, from the mean flame surface. As described previously, the solutions for the mixture fraction fields required to evaluate this expression must generally be obtained from the computations. However, to aid in the interpretation of these results, it is helpful to consider the analytical solution using the step inlet boundary condition given by Eq.(13), derived previously by Magina et al. [29] in the Pe > 1 limit for axial bulk forcing:

$$
\frac{\xi_{1, n}(x, t)}{R_{f}}=-\left[\frac{\varepsilon \sin \theta_{0}(x) \exp (-i \omega t)}{2 \pi i S t_{R}}\right]\left[1-\exp \left(\frac{2 \pi i f x}{U_{0}}\right) \exp \left(-\frac{4 \pi^{2} S t_{R}^{2}}{P e} \frac{x}{R_{f}}\right)\right]+O\left(\frac{1}{P e^{2}}\right)
$$

Inside the waveform term shown by the second set of brackets, the first unity term stems from the particular solution. It lacks spatial dependence due to the nature of the imposed bulk forcing. The second term describes a traveling wave generated at the fuel port lip, $x=0$. This expression shows that flame wrinkles convect at the mean flow velocity, $U_{0}$, without dissipation in the $P e \rightarrow \infty$ limit, a feature which stems from the assumed $Z_{1}=0$ inflow (and implied attachment) condition. Local maxima and minima in flame wrinkle amplitude arise through the interference between the two terms. For example, nodes in flame wrinkling amplitude occur at $x=n U_{0} / f$ where $n$ is a positive integer. These trends can be clearly seen by re-writing this bracketed term in the $P e \rightarrow \infty$ limit as:

$$
1-\exp \left(i 2 \pi f \frac{x}{U_{0}}\right)=2 \sin \left(\pi f \frac{x}{U_{0}}\right) \exp \left(i\left[\pi f \frac{x}{U_{0}}-\frac{\pi}{2}\right]\right)
$$

The $\mathrm{O}(1 / P e)$ terms cause flame surface wrinkles to decay exponentially with downstream distance. This causes the interference effect discussed above to become imperfect, an effect which increases quadratically with $S t$. The mechanism for wave dissipation is due to the progressive smoothing of the spatial variations in the mixture fraction field by diffusion with downstream distance.

Figure 6 plots the axial dependence of the flame response magnitude, $\left|\xi_{1}\right|$, for the $P e \rightarrow \infty$ analytical solutions, along with various extended inlet numerically computed results, for various values of $P e S t_{R}$. Comparing results at fixed values of this non-dimensional parameter allows for the demonstration of the effect of axial diffusion, by keeping the number of wrinkles on the flame length constant. The plots incorporating axial diffusion have similar trends, however, the smoothing of the node/anti-node behavior discussed previously is clearly evident from the reduced peaks and nodes, resulting from imperfect interference between the waveform terms. This effect becomes more pronounced at higher forcing frequencies, where more spatial wrinkles exist, and also at larger axial distances 
downstream. Note also that the magnitude of flame movement is nonzero at $x=0$ in the smaller Peclet number cases, as explained in Section 3.2.

\subsection{Spatially Integrated Heat Release}

The spatially integrated quantity is of particular interest for combustion instability or noise related issues where the flame is small relative to the acoustic wavelength [1]. Thus, next consider the spatially integrated heat release, $\dot{Q}(t)$, given by the following surface integral over the reaction sheet (obtained by relating species mass fractions to mixture fraction) [28]:

$$
\dot{Q}(t)=\int_{\text {flame }} \frac{\left(1+\varphi_{o x}\right)^{2}}{\varphi_{o x}} \rho O S h_{R}\left|\frac{\partial Z}{\partial n}\right| d A
$$

The instantaneous global heat release of the flame, given by Eq.(20), can be linearized and written in terms of the perturbations, where fixed composition fuel and oxidizer is assumed so that the heat of reaction and mixture density are constant. Consequently, heat release oscillations are induced by fluctuations in mass burning rate and flame surface area. An expression for the linearized, instantaneous spatially integrated heat release is [29, 33]:

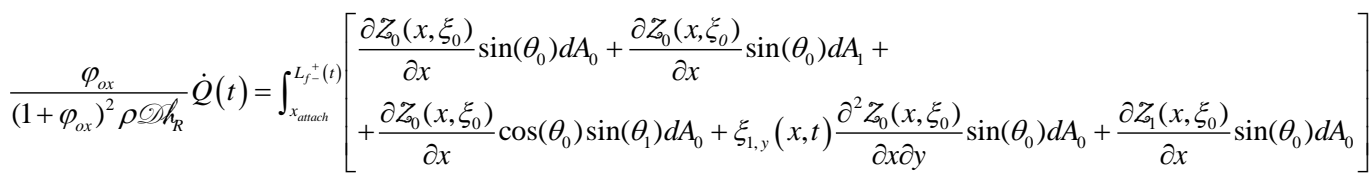

$$
\begin{aligned}
& -\int_{x_{\text {atanct }}}^{L_{f^{+}(t)}}\left[\begin{array}{l}
\underbrace{\frac{\partial Z_{0}\left(x, \xi_{0}\right)}{\partial y} \cos \left(\theta_{0}\right) d A_{0}}_{q_{0}(x)}+\underbrace{\frac{\partial Z_{0}\left(x, \xi_{0}\right)}{\partial y} \cos \left(\theta_{0}\right) d A_{1}}_{q_{A}(x, t)}+ \\
-\underbrace{\frac{\partial Z_{0}\left(x, \xi_{0}\right)}{\partial y} \sin \left(\theta_{0}\right) \sin \left(\theta_{1}\right) d A_{0}+\xi_{1, y}(x, t) \frac{\partial^{2} Z_{0}\left(x, \xi_{0}\right)}{\partial y^{2}} \cos \left(\theta_{0}\right) d A_{0}+\frac{\partial Z_{1}\left(x, \xi_{0}\right)}{\partial y} \cos \left(\theta_{0}\right) d A_{0}}_{q_{M B R}(x, t)}
\end{array}\right]
\end{aligned}
$$

where in a rectangular coordinate system, the differential areas can be written in multiple ways, depending upon whether the integration is performed over the axial or transverse coordinate; e.g.,:

$$
d A_{1}=\frac{\sin \theta_{0} \sin \theta_{1}}{\cos ^{2} \theta_{0}} d x \quad d A_{1}=\frac{-\cos \theta_{0} \sin \theta_{1}}{\sin ^{2} \theta_{0}} d y
$$

This integral is evaluated from the flame base attachment point, $x_{\text {attach }}$, to the flame tip via both the top, $L_{f}^{+}(t)$, and bottom, $L_{f}^{-}(t)$, flame branches. This expression has been written in this specific form in order to isolate the various contributing mechanisms to the heat release. The brackets separate contributions from included transverse (first set) and axial (second set) diffusion, i.e. the $d z / d y$ and $d z / d x$ terms in Eq.(20), respectively. Within each set of brackets, the first and second terms denote the steady state contribution and the contribution due to area fluctuations, respectively, while the remaining terms indicate the contributions due to mass burning rate fluctuations. These mass burning rate fluctuations arise due to the fluctuations in spatial gradients of the mixture fraction. The next few sections analyze various important features of this equation. The distributions of the mean and fluctuating heat release will be investigated along with how they influence the heat release transfer function trends and asymptotics.

\subsubsection{Distribution of the mean and fluctuating heat release}

In two-dimensional premixed flames, the axial distribution of heat release does not vary strongly, as the mass burning rate per unit area is directly proportional to the laminar burning velocity, which is not usually a strong function of the axial position. For this reason, premixed flame heat release oscillations are closely correlated with oscillations in flame surface area. In contrast, the axial distribution of heat release in non-premixed flames can vary by several orders of magnitude, as the strong transverse gradients that drive fuel/oxidizer flux near the burner outlet are smoothed with downstream distance. To illustrate, Figure 7 shows the steady state distribution of heat release per 
unit area, $q_{0}(x)$, along the flame sheet, for various $P e$ values. The cumulative heat release distribution, $q_{0, \mathrm{c}}$, is also plotted, defined as:

$$
q_{0, c}(x)=\frac{\int_{0}^{x} q_{0}(x) d x}{Q_{0}}
$$

where $Q_{0}$ is the steady state heat release defined as, $Q_{0}=\int_{0}^{L_{f, 0}} q_{0}(x) d x$. Also plotted for reference is the axial distribution for a constant burning velocity premixed flame, indicated by "PM Flame" in the legend.

For the steady state distribution, the $P e \rightarrow \infty$ limiting case has an integrable singularity at the inlet that can be shown analytically to scale as $\dot{m}_{F}^{\prime \prime} \sim x^{-1 / 2}$, as also shown in Figure 7 . With finite $P e$ values, the inlet profile is smoothed and the peak value at $x=0$ is reduced. In addition, the heat release contribution from the tip increases with decreasing $P e$ value. Comparing and contrasting the premixed and non-premixed flame plots shows that half of the average heat release occurs in roughly the first $15-20 \%$ of the non-premixed flame, while it occurs at the $50 \%$ flame midpoint for the premixed flame. This result clearly shows the need for particular care in accounting for inflow conditions that influence the $x / R_{f}<<1$ region for non-premixed flame problems.

Figure 8 shows the corresponding frequency domain representation of the unsteady heat release distribution, $\hat{q}_{1}(x)$, for various $P e$ and $S t$ values. The cumulative heat release distribution is plotted in Figure 9 with two different normalizations, defined as:

$$
q_{1, c_{1}}=\frac{\int_{0}^{x} \hat{q}_{1}(x) d x}{\varepsilon Q_{0}} \quad q_{1, c_{2}}=\frac{\int_{0}^{x} \hat{q}_{1}(x) d x}{\int_{0}^{L_{f, 0}} \hat{q}_{1}(x) d x}
$$

The first definition uses the steady state heat release for normalization so that its value at $x / L_{f, 0}=1$ corresponds to the flame transfer function's value (discussed later). The second normalization uses the spatially integrated unsteady heat release, so that its value at $x / L_{f, 0}=1$ goes to unity, thus allowing one to visualize which parts of the flame contribute to its unsteady heat release. Figure 8 shows that, for $P e>>1$ and $S t<<1$, the largest values of local fluctuating heat release occur at both the flame base, due to the sharp inlet gradient, and at the flame tip, due to the pulsing flame length. However, neither of these regions contributions to the cumulative heat release are dominant, as shown in Figure 9.

For $P e \gg>1$ and $S t \sim \mathrm{O}(1)$ or $\gg>1$, the local heat release at the tip diminishes, and the largest local values occur at the flame base. Moreover, the cumulative distributions show that the majority of the unsteady heat release occurs at the flame base - a fact which enables the development of simple asymptotic expressions for the $S t>>1$ heat release, to be discussed in the next section. The same $S t$ trends hold for lower finite $P e$ values, yet the base and tip (for low $S t$ values), contributions are reduced and the middle length of flame has a larger contribution due to axial diffusion.

\subsubsection{Heat release transfer functions - General Results}

Having considered the spatial distributions of the heat release, we next consider its spatially integrated value. The flame transfer function, $\mathscr{F}$, is a useful parameter which quantifies the input-output relation between forcing and heat release, is defined as:

$$
\mathscr{F}=\frac{\hat{\dot{Q}}_{1} / \dot{Q}_{0}}{\hat{u}_{1} / U_{0}}
$$

Figure 10 plots the computed amplitude and phase of $\mathscr{F}$ as a function of $S t$ for various $P e$ values, utilizing Eq.(21), as well as the $P e \rightarrow \infty$ result previously obtained [29]. For the amplitude, the most prominent difference between the results which include and neglect axial diffusion are the asymptotic characteristics; the $P e \rightarrow \infty$ result having a two-zone structure, while the results with axial diffusion show a three zone asymptotic structure. These asymptotic roll off values, along with the transitional $S t$ values (some of which are dependent upon $P e$ ) will be focused upon later in this section. The phase plot also shows some differences between the results, although they all appear qualitatively similar. 
The $P e \rightarrow \infty$ transfer function curve and corresponding high $S t$ asymptotics were discussed in our prior study [29], and are summarized below for context in order to understand finite $P e$ effects. For the axially forced configuration considered in this study, in the $P e \rightarrow \infty$ limit, two important simplifications can be made; first, only the $\partial Z / \partial y \cos \theta$ terms in Eq.(21) remain, and second, the area contribution, in Eq.(21), can be analytically shown to cancel out the first mass burning rate term, resulting in [29]:

$$
P e \rightarrow \infty \quad \frac{-\varphi_{o x}}{\left(1+\varphi_{o x}\right)^{2} \rho \partial \partial h_{R}} \dot{Q}_{1}(t)=\int_{0}^{L_{f, 0}{ }^{+}}\left[\xi_{1, n}(x, t) \frac{\partial^{2} \xi_{0}\left(x, \xi_{0}(x)\right)}{\partial y^{2}}+\frac{\partial Z_{1}\left(x, \xi_{0}(x)\right)}{\partial y}\right] d x
$$

By utilizing expressions for mixture fraction and fluctuating flame position, from Sections 3.1 and 3.3 respectively, this transfer function can be alternatively expressed as:

$$
P e \rightarrow \infty \quad \mathscr{F}=\frac{\int_{0}^{L_{f, 0} 0^{ \pm}}\left[\frac{-i R_{f}}{2 \pi S t_{R}}\right] g\left(x, \xi_{0}\right)\left\{1-\exp \left(2 \pi i S t_{R} \frac{x}{R_{f}}\right)\right\} d x}{\int_{0}^{L_{f, 0}{ }^{ \pm}} \frac{\partial Z_{0}\left(x, \xi_{0}\right)}{\partial y} d x}
$$

where $g\left(x, \xi_{0}\right)$ is an axial distribution function, which will be utilized extensively in the next sections due to its influence over the characteristics of this oscillatory integral. For the $P e \rightarrow \infty$ case, this term is defined as:

$$
g\left(x, \xi_{0}\right)=\tan \left(\theta_{0}\right) \frac{\partial^{2} Z_{0}\left(x, \xi_{0}\right)}{\partial y^{2}}+\frac{d^{2} z_{0}\left(x, \xi_{0}\right)}{d x d y}
$$

\subsubsection{Heat release transfer functions - Low Strouhal Asymptotic Results}

Expanding $\mathscr{F}$, as given by Eq.(27), in powers of $S t$ (see Appendix B), leads to:

$$
S t \ll 1 \quad \mathscr{F}=1+i S t_{L f} \frac{2 \pi \int_{0}^{L_{f, 0}} x \frac{\partial Z_{0}\left(x, \xi_{0}(x)\right)}{\partial y} \cdot d x}{L_{f, 0} \int_{0}^{L_{f, 0}} \frac{\partial \mathcal{Z}_{0}\left(x, \xi_{0}(x)\right)}{\partial y} \cdot d x}+O\left(S t_{L f}^{2}\right)
$$

Thus, $|\mathscr{F}|=1+\mathrm{O}\left(S t^{2}\right)$ for $S t<<1$. This value of unity can be understood physically from the fact that in the quasisteady limit, the instantaneous heat release is proportional to the instantaneous mass flow rate of fuel and oxidizer into the combustion domain, i.e. across the inlet plane. The low $S t$ phase behavior can also be extracted from this expression, since $\angle \mathscr{F}=\tan ^{-1}(\operatorname{Im}(\mathscr{F}) / \operatorname{Re}(\mathscr{F}))$. This can then be cast in terms of a $n$ - $\tau$ model as [35]:

$$
\text { St } \ll<1 \quad \mathscr{F}=\eta \exp [i \omega \tau]
$$

or likewise, represented in the time domain as:

$$
S t \ll 1 \quad \frac{\dot{Q}_{1}}{\dot{Q}_{0}}=\eta \frac{u_{1}(t-\tau)}{U_{0}}
$$

where the gain is unity, i.e. $\eta=1$, and the time delay is given by:

$$
\tau=\left[\frac{\int_{0}^{L_{f, 0}} x \frac{\partial Z_{0}\left(x, \xi_{0}(x)\right)}{\partial y} \cdot d x}{L_{f, 0} \int_{0}^{L_{f, 0}} \frac{\partial Z_{0}\left(x, \xi_{0}(x)\right)}{\partial y} \cdot d x}\right] \frac{L_{f, 0}}{U_{0}}
$$


This time delay is equivalent to replacing the distributed flame by a concentrated source at some fractional distance of the flame length. While the gain is straightforward, the time delay is a complex function of parameters such as $P e$ and $Z_{s t}$. These parametric dependencies of $\tau$ cannot be calculated analytically but must be extracted from the computations, once again due to the implicit nature of the mean flame position at which these integrand values are evaluated. This value (normalized by $L_{f, 0} / U_{0}$ ), for example, is around 0.2 for a methane-air, non-premixed system, which has a $Z_{s t}$ value of 0.055 . For reference, the constant burning velocity two-dimensional bulk axially forced premixed flame result is $\tau /\left(L_{f, 0} / U_{0}\right) \approx 0.5$ (assuming the flow velocity is much greater than the flame speed), showing that the non-premixed flame time delay is about a factor of two smaller than a premixed flame with the same length. This result directly follows from the heat release distributions shown in Figure 7.

Figure 11 plots calculations of $\tau /\left(L_{f, 0} / U_{0}\right)$ as a function of $P e$ for several $Z_{s t}$ values, representative of positive and negative (nomenclature defined in Figure 4) fuel port attachment location diffusion flame configurations. The corresponding premixed flame value is also plotted for reference. The solid lines indicate the numerical extended inlet results, while the dashed lines show the corresponding $P e \rightarrow \infty$ analytical results, given by Eq.(32).

Convergence at high $P e$ values was validating, while the deviation of the asymptotic results from the computed ones for lower $P e$ values is due to both axial diffusion effects upon this effective time delay, but also reflects the importance of how flame length is defined; i.e., the flames base moves upstream into the fuel/oxidizer ports with decreasing $P e$, see Figure 3, while $L_{f, 0}$ used in the figure is defined as the distance of the flame tip from the burner outlet.

As noted in the context of Eq.(21), the heat release fluctuations can be decomposed into contributions associated with burning rate and flame area. However, non-premixed flame heat release oscillations are dominated by mass flux oscillations near the burner outlet, where these gradients are the sharpest. The flame surface area oscillations have little influence on the overall heat release because the local burning rate is so low over most of the flame. In the absence of axial diffusion, the ratio of mass burning rate to area contributions from the unsteady heat release can be shown to be of order $\mathrm{O}\left(\mathrm{Pe}^{2}\right)$ in the low St limit. More generally, Figure 12 plots the ratio of the magnitudes of the spatially integrated mass burning rate and area terms, for a representative low $S t$ value, as a function of $P e$, defined as:

$$
Q_{M B R}=\int_{0}^{L_{f, 0}} \hat{q}_{M B R}(x) d x \quad Q_{A}=\int_{0}^{L_{f, 0}} \hat{q}_{A}(x) d x
$$

where $\hat{q}_{M B R}$ and $\hat{q}_{A}$ were previously denoted in Eq.(21). Note the excellent agreement of the computed and $P e \rightarrow \infty$ results at large $P e$ values, both with each other and to the analytically obtained scaling. At higher Strouhal numbers, the mass burning rate continues to dominate relative to area fluctuations, but has a more complex dependency on $S t$ and $P e$.

\subsubsection{Heat release transfer functions - High Strouhal Asymptotic Results}

Having considered the low $S t$ results, we next consider higher frequency transfer function characteristics. As was previously shown by the cumulative unsteady heat release distribution results in Figure 9, in this limit the unsteady heat release is dominated by its value at $x / L_{f, 0}<<1$. It is common for highly oscillatory integrals, similar in form to Eq. (27), to be controlled by the values of the integrand at the boundaries (i.e. $x=0$ and $x=L_{f, 0}$ ), and generally lead to a $1 / S t$ asymptotic behavior. However, as is shown in Figure 10, the transfer function has a $1 /\left(S t^{1 / 2}\right)$ behavior for the $P e \rightarrow \infty$ case, and only for finite $P e$ values does it transition to $1 / S t$ at some sufficiently high $S t$.

For a step inflow profile, the mass burning rate has an integrable singularity at the $x=0$ inlet boundary in the $P e \rightarrow \infty$ limit, a singularity which controls the heat release asymptotics. It was shown previously that the $S t>>1$ transfer function is given by [29]:

$$
P e \rightarrow \infty \quad \mathscr{F} \approx-(1+i) /\left(4 \sqrt{S t_{L_{f}}}\right)
$$

i.e., $|\mathscr{F}| \sim 1 /\left(S t^{1 / 2}\right)$, as indicated in Figure 10. Since we have shown the flame transfer function amplitude is unity in the limit of low $S t$ values, the Strouhal number at which this first transition occurs, from $\mathrm{O}(1)$ to $\mathrm{O}\left(S t^{-1 / 2}\right)$, will be denoted $S t_{1}$ and can be obtained from the following expression, representing the intersection of limits: 


$$
\left|\frac{i+1}{4 \sqrt{S t_{1}}}\right|=1 \quad \longrightarrow \quad S t_{1}=\frac{1}{8}
$$

Even for more realistic inflow profiles that are smooth but very thin relative to the convective wavelength at $x=0$ (i.e., the finite $P e$ case), $|\mathscr{F}|$ still has a $1 / S t^{1 / 2}$ character, for the same reasons as the step inlet, for some range of $S t$ values. However, for disturbances with length scales that are on the order of the inflow profile thickness, different asymptotic considerations apply which ultimately cause the non-premixed flame to exhibit a $1 / S t$ asymptotic behavior that one would expect of highly oscillatory integrals.

At this point, it is important that we differentiate and isolate the various effects of axial diffusion on the heat release fluctuations, i.e., inflow boundary effects and dynamical effects. By "dynamical effects", we are referring to the inclusion of axial diffusion in the unsteady mixture fraction equation, i.e. setting $b=1$ in Eq.(4). These effects upon the space-time dynamics were noted previously in Section 3.3, resulting in dissipation and dispersion of flame wrinkles. By "inflow boundary effects", we are referring to the inclusion of axial diffusion in the steady mixture fraction equation, i.e., by setting $b=1$ in Eq.(3), effects previously discussed in Section 3.1.

These two effects can be isolated and the resulting transfer function magnitudes are plotted in Figure 13. These results were generated by repeating the calculations and selectively including or neglecting axial diffusion in the steady and unsteady mixture fraction equations. To isolate the dynamical effects, axial diffusion was not included in the steady governing equation, thus retaining the sharp step inlet boundary condition, and was included in the dynamical fluctuating equation; this solution is indicated by " $Z_{0}: P e \rightarrow \infty \& Z_{1}: P e$ finite" in the figure. To isolate the inlet effects, axial diffusion was included in the steady governing equation, allowing the smoothing of the inlet profile, and was excluded from the dynamical fluctuating equation, denoted as " $Z_{0}: P e$ finite $\& Z_{1}: P e \rightarrow \infty$ " in the figure. Also shown are cases where axial diffusion is excluded in both equations and included in both.

The figure clearly shows that it is the smoothed inflow profile that controls the high $S t$ asymptotic features. In other words, the key effect of axial diffusion on the asymptotic characteristics of $\mathscr{F}$ is on the time averaged profiles of $z$, specifically the inlet profile - i.e., axial diffusion effects on the unsteady dynamics of $z$ have minor influences on $\mathscr{F}$, although they have important influences on the downstream evolution of the flame position, as shown by Eq.(18).

To demonstrate this point explicitly, it is useful to consider solution properties of a model problem with a finite thickness $Z_{0}$ transition layer, parameterized by $\delta$, shown in Figure 14. In reality, the non-dimensional profile thickness parameter, $\delta$, simulates the extent to which axial diffusion has altered the inlet boundary condition, and is proportional to the inverse of $P e$. In this model problem, however, we decouple this dependence of $\delta$ upon $P e$.

Utilizing this inflow boundary condition for the unconfined system, and the governing equation given by Eq.(3), with $b=0$ (thus isolating the inflow boundary effect), the full steady state mixture fraction solution can be solved and is provided in Appendix C. In order to make analytical progress, we consider the limiting characteristics of this solution for large, but finite, $P e$ values (small $\delta$ values) and small axial locations. The latter limit is of interest as the majority of unsteady heat release occurs near the burner lip, as was shown in Figure 7 and Figure 9. This simplified mixture fraction solution is shown below:

$$
\underset{x / P e R_{f}<<1}{\&} Z_{0}(x, y)=\frac{-1}{4 \delta}\left[\begin{array}{l}
\exp \left(-\frac{\left(-1+y / R_{f}\right)^{2}}{4 x /\left(P e R_{f}\right)}\right)\left[2 x /\left(P e R_{f}\right)+\frac{4 \sqrt{x /\left(P e R_{f}\right)}}{\sqrt{\pi}}\right]\left\{\frac{\delta\left(-1+y / R_{f}\right)}{x /\left(P e R_{f}\right)}\right\}+ \\
-\exp \left(-\frac{\left(1+y / R_{f}\right)^{2}}{4 x /\left(P e R_{f}\right)}\right)\left[2 x /\left(P e R_{f}\right)+\frac{4 \sqrt{x /\left(P e R_{f}\right)}}{\sqrt{\pi}}\right]\left\{\frac{\delta\left(1+y / R_{f}\right)}{x /\left(P e R_{f}\right)}\right\}+ \\
-\sqrt{P e\left(\delta+1-y / R_{f}\right)^{2}}-\sqrt{P e\left(\delta+1+y / R_{f}\right)^{2}}+\sqrt{P e\left(\delta-1-y / R_{f}\right)^{2}}+\sqrt{P e\left(\delta-1+y / R_{f}\right)^{2}}
\end{array}\right]
$$

Since as we discussed, the key effect of axial diffusion on the asymptotic characteristics of $\mathscr{F}$ is on the time averaged profiles of $Z$, rather than the unsteady dynamics, it is useful to consider the $P e \rightarrow \infty$ solution shown in Eq.(27) utilizing these smoothed, time average inflow profiles. Inclusion of finite $P e$ effects in the solution for the flame position, such as dissipation, into the heat release transfer function shown in Eqn.(27) would not alter these high $S t$ asymptotics. This is because in the high $S t$ limit all the exponential terms tend towards zero and it is the unity 
constant that results in the highest $S t$ asymptotic region rolling off as $1 / S t$. Further exponential terms, such as $\exp (-$ $4 \pi^{2} S t_{\mathrm{R}}{ }^{2} * x / P e^{*} R_{f}$ ), would simply drive this term to zero sooner. Thus, this model problem simulates the smoothing effect of axial diffusion on the inlet boundary condition, eliminating the singularity caused by the step inlet boundary condition, while maintaining the $P e \rightarrow \infty$ limit for the unsteady governing equation. Returning to Eq.(27), the function $g\left(x, \xi_{0}\right)$ is given for the smoothed profile as:

$$
g\left(x, \xi_{0}\right)=\frac{\sqrt{P e}}{2 x^{3 / 2} \sqrt{R_{f} \pi}}+\frac{1}{4 \delta x}
$$

Substituting this expression into Eq.(27) shows that in the limit of large $S t$ :

$$
P e \rightarrow \infty, S t>>1 \quad \mathscr{F}=\frac{C_{1}}{\sqrt{S t_{L f}}}+\frac{C_{2}}{S t_{L f}} \frac{1}{P e \delta} \sqrt{\frac{P e L_{f}}{R_{f}}}
$$

where

$$
C_{1}=\frac{(i+1)}{4} \quad C_{2}=\frac{i[\lambda-4 \sqrt{\pi}]}{16 \sqrt{\pi}}
$$

and $\lambda$ is Euler's constant, with numerical value $\lambda \square 0.577216$. Note how the leading order $S t>>1$ term is $\mathrm{O}\left(S t^{-1 / 2}\right)$, while the next term is of $\mathrm{O}\left(S t^{-1}\right)$. Thus, while a $S t^{-1 / 2}$ behavior occurs for the step function exit profiles shown in Eq.(13), a smooth mixture fraction at the base of the flame leads to the $1 / S t$ asymptotic behavior that one would expect of highly oscillatory integrals. For this model problem we can develop an explicit expression for the Strouhal number at which $S t^{-1 / 2}$ to $S t^{-1}$ transition occurs, denoted as $S t_{2}$, by equating these two terms:

$$
\left|\frac{C_{1}}{\sqrt{S t_{2}}}\right|=\left|\frac{C_{2}}{S t_{2}} \frac{1}{P e \delta} \sqrt{\frac{P e L_{f}}{R_{f}}}\right| \quad \longrightarrow \quad S t_{2}=\left(\frac{\left|C_{2}\right|}{\left|C_{1}\right|}\right)^{2} \frac{1}{P e^{2} \delta^{2}} \frac{P e L_{f}}{R_{f}}
$$

Physically, this transition Strouhal number corresponds with conditions where the convective disturbance wavelength is on the order of the axial extent required for fuel/oxidizer to diffuse across the inlet transition layer, i.e. $U_{0} / f \sim U_{0}\left(R_{f} \delta\right)^{2} / \mathscr{D}$. In reality, $P e$ and $\delta$ are not independent, and if we substitute in $\delta \sim 1 / P e$, this shows that:

$$
S t_{2}=\left(\frac{\left|C_{2}\right|}{\left|C_{1}\right|}\right)^{2} \frac{P e L_{f}}{R_{f}}
$$

Armed with this insight from the model problem, we return to the computed results. These results show that three different $S t$ ranges exist, each with a well defined $S t$ roll off; transitioning from $S t^{0}$ roll off, at low $S t$ values, to $1 /\left(S t^{1 / 2}\right)$, for intermediate $S t$ values, to $1 / S t$, for high $S t$ values. As $S t_{2}$ is a function of $P e$ and $L_{f}$, these transition points are more easily observed by rescaling the axes. Figure 15 replots the data shown in Figure 10 by normalizing the horizontal axis by $S t_{2}$ and the vertical axis by $\mathrm{C}_{1} /\left(S t_{2}\right)^{1 / 2}$. The figure shows how this scaling collapses the high $S t$ results at the different $P e$ values.

\section{Conclusions}

This paper focuses on axial diffusion effects on axially forced non-premixed flame systems, and the influence this additional physics has on the space-time and heat release dynamics. The interesting spatial dynamical features were shown to be attributed to either dynamical or inlet effects. Although several approximate forms exist in the literature, it was shown that in general, the inlet mixture fraction profile had to be determined computationally and 
extracted from a numerical solution which simultaneously takes into account both the fuel/oxidizer port $(x<0)$ and combustion zone $(x>0)$ domains. Compared to the commonly utilized step-inlet condition, axial diffusion smoothes the inlet profile and as a resultant effect, the instantaneous flame attachment point oscillates axially, as opposed to remaining fixed at the fuel port lip. As for dynamical effects resulting from the inclusion of axial diffusion into the governing $z$-equation, computations reveal that axial diffusion introduces a smoothing mechanism for flame wrinkles as they move downstream, eliminating spatially nodes that occur in the $P e \rightarrow \infty$ limit. This effect can be verified analytically when $\mathrm{O}\left(P e^{-1}\right)$ terms are included at large, yet finite $P e$ values for simplified mixture fraction field solutions. Lastly, axial diffusion was shown to have a profound influence upon the local heat release distribution, as well as the global heat release asymptotics. An $n-\tau$ model was obtained for the low $S t$ asymptotics of the heat release transfer functions, while axial diffusion was shown to alter the corresponding high $S t$ asymptotics, producing an additional $S t$ roll off region of $1 / S t$ at large values. This effect, which was elucidated analytically with a simplified model problem, was shown to be a result of the smoothing action of the mixture fraction gradient at the fuel port lip.

\section{Acknowledgments}

This work has been partially supported by the National Science Foundation under contract CBET-1235779, contract monitor Professor Ruey-Hung Chen, and by the US Department of Energy's University Turbine Systems Research Program under contracts DE-FE0025174, contract monitor Seth Lawson. 


\section{References}

[1] T.C. Lieuwen, Unsteady Combustor Physics. 2012: Cambridge University Press.

[2] T. Lieuwen, Modeling premixed combustion-acoustic wave interactions: A review. Journal of Propulsion and Power, 2003. 19(5): p. 765-781.

[3] F. Baillot, D. Durox, R. Prud'Homme, Experimental and theoretical study of a premixed vibrating flame. Combustion and flame, 1992. 88(2): p. 149-152, IN1, 153-168.

[4] Y. Huang, V. Yang, Dynamics and Stability of Lean-Premixed Swirl-Stabilized Combustion. Progress in Energy and Combustion Science, 2009. 35: p. 293-364.

[5] S.H. Preetham, T. Lieuwen, Dynamics of laminar premixed flames forced by harmonic velocity disturbances. Journal of Propulsion and Power, 2008. 24(6): p. 1390-1402.

[6] T. Schuller, D. Durox, S. Candel, A unified model for the prediction of laminar flame transfer functions: comparisons between conical and V-flame dynamics. Combustion and Flame, 2003. 134(1-2): p. 21-34.

[7] L. Boyer, J. Quinard, On the dynamics of anchored flames. Combustion and flame, 1990. 82(1): p. 51-65.

[8] M. Fleifil, A.M. Annaswamy, Z.A. Ghoneim, A.F. Ghoneim, Response of a laminar premixed flame to flow oscillations: A kinematic model and thermoacoustic instability results. Combustion and flame, 1996. 106: p. 487510.

[9] D. Chamberlin, A. Rose, The flicker of luminous flames. Industrial \& Engineering Chemistry, 1928. 20(10): p. 1013-1016.

[10] J. Buckmaster, N. Peters. The infinite candle and its stability-a paradigm for flickering diffusion flames. in Symposium (International) on Combustion. 1988: Elsevier.

[11] J. Boulanger, Laminar round jet diffusion flame buoyant instabilities: Study on the disappearance of varicose structures at ultra-low Froude number. Combustion and flame, 2010. 157(4): p. 757-768.

[12] L.-W. Chen, Q. Wang, Y. Zhang, Flow characterisation of diffusion flame under non-resonant acoustic excitation. Experimental Thermal and Fluid Science, 2013. 45: p. 227-233.

[13] H.W. Huang, Q. Wang, H.J. Tang, M. Zhu, Y. Zhang, Characterisation of external acoustic excitation on diffusion flames using digital colour image processing. Fuel, 2012. 94: p. 102-109.

[14] Q. Wang, H.W. Huang, H.J. Tang, M. Zhu, Y. Zhang, Nonlinear response of buoyant diffusion flame under acoustic excitation. Fuel, 2013. 103: p. 364-372.

[15] R. Chrystie, S.H. Chung, Response to Acoustic Forcing of Laminar Coflow Jet Diffusion Flames. Combustion Science and Technology, 2014. 186(4-5): p. 409-420.

[16] T.C. Williams, C.R. Shaddix, R.W. Schefer, P. Desgroux, The response of buoyant laminar diffusion flames to low-frequency forcing. Combustion and Flame, 2007. 151(4): p. 676-684.

[17] M. Saito, M. Sato, A. Nishimura, Soot suppression by acoustic oscillated combustion. Fuel, 1998. 77(9): p. 973-978.

[18] D. Ferreira, P. Lacava, M. Ferreira, J. de Carvalho, JA, NOx and CO emissions and soot presence in partially premixed acoustically excited flames. Journal of the Energy Institute, 2009. 82(3): p. 123-132.

[19] R.K. Mohammed, M.A. Tanoff, M.D. Smooke, A.M. Schaffer, M.B. Long. Computational and experimental study of a forced, timevarying, axisymmetric, laminar diffusion flame. in Symposium (International) on Combustion. 1998: Elsevier.

[20] S. Dworkin, B. Connelly, A. Schaffer, B. Bennett, M. Long, M. Smooke, M. Puccio, B. McAndrews, J. Miller, Computational and experimental study of a forced, time-dependent, methane-air coflow diffusion flame. Proceedings of the Combustion Institute, 2007. 31(1): p. 971-978.

[21] M. Tyagi, N. Jamadar, S. Chakravarthy, Oscillatory response of an idealized two-dimensional diffusion flame: Analytical and numerical study. Combustion and flame, 2007. 149(3): p. 271-285.

[22] S. Chung, C. Law, Burke-Schumann flame with streamwise and preferential diffusion. Combustion Science and Technology, 1984. 37(1-2): p. 21-46.

[23] C. Law, S. Chung, Steady state diffusion flame structure with Lewis number variations. Combustion Science and Technology, 1982. 29(3-6): p. 129-145.

[24] K. Balasubramanian, R. Sujith, Nonlinear response of diffusion flames to uniform velocity disturbances. Combustion science and technology, 2008. 180(1-3): p. 418-436.

[25] K. Balasubramanian, R. Sujith, Non-normality and nonlinearity in combustion-acoustic interaction in diffusion flames. Journal of Fluid Mechanics, 2008. 594: p. 29-58.

[26] M. Tyagi, S. Chakravarthy, R. Sujith, Unsteady combustion response of a ducted non-premixed flame and acoustic coupling. Combustion Theory and Modelling, 2007. 11(2): p. 205-226. 
[27] L. Magri, K. Balasubramanian, R. Sujith, M.P. Juniper, Non-normality in combustion-acoustic interaction in diffusion flames: a critical revision. arXiv preprint arXiv:1310.0238, 2013.

[28] N. Magina, D.-H. Shin, V. Acharya, T. Lieuwen, Response of non-premixed flames to bulk flow perturbations. Proceedings of the Combustion Institute, 2013. 34(1): p. 963-971.

[29] N. Magina, V. Acharya, T. Sun, T. Lieuwen, Propagation, dissipation, and dispersion of disturbances on harmonically forced, non-premixed flames. Proceedings of the Combustion Institute, 2015. 35(1): p. 1097-1105.

[30] N. Magina, V. Acharya, T. Lieuwen, Response of non-premixed flames to bulk flow perturbations. Eastern States Section of the Combustion Institute, 2011.

[31] N. Magina, T. Lieuwen, Response of over-ventilated non-premixed flames to transverse flow perturbations. Eastern States Section of the Combustion Institute, 2013.

[32] Y. Xiong, M.S. Cha, S.H. Chung, Fuel density effect on near nozzle flow field in small laminar coflow diffusion flames. Proceedings of the Combustion Institute, 2015. 35(1): p. 873-880.

[33] N.A. Magina, T.C. Lieuwen. Effect of axial diffusion on the response of over-ventilated diffusion flames to axial flow perturbations. in 52nd Aerospace Science Meeting. 2014.

[34] F.A. Williams, Combustion theory. 2nd ed. 1985, Menlo Park, Ca: Benjamin/Cummings Publishing Co.

[35] L. Crocco, S.-I. Cheng, Theory of combustion instability in liquid propellant rocket motors. 1956: Cambridge Univ Press. 


\section{Appendix A}

Insight regarding this claim, and the condition at $x=0$ can be attained by integrating Eq.(1) from the reservoir plane at $x=-\infty$, where the mixture fraction values are set and constant, to the inflow plane $x=0$. This yields:

$$
\int_{-\infty}^{0} \frac{\partial \mathscr{Z}}{\partial t} d x+\left.u_{x} Z\right|_{x=0}=\left.u_{x} Z\right|_{x=-\infty}+\mathscr{O} \int_{-\infty}^{0} \frac{\partial^{2} Z}{\partial y^{2}} d x+\left.b \mathscr{O} \frac{\partial \mathscr{Z}}{\partial x}\right|_{x=0}
$$

where $u_{x}$ is the instantaneous axial velocity. Expanding Eq.(42) into nominal and fluctuating values, and employing the reservoir conditions leads to:

$$
\begin{gathered}
\left.U_{0} Z_{0}\right|_{x=0}=\left.U_{0} Z_{0}\right|_{x=-\infty}+\mathscr{O} \int_{-\infty}^{0} \frac{\partial^{2} Z_{0}}{\partial y^{2}} d x+\left.b \mathscr{O} \frac{\partial Z_{0}}{\partial x}\right|_{x=0} \\
\int_{-\infty}^{0} \frac{\partial Z_{1}}{\partial t} d x+\left.U_{0} Z_{1}\right|_{x=0}+\left.u_{1} Z_{0}\right|_{x=0}=\left.u_{1} Z_{0}\right|_{x=-\infty}+\mathscr{O} \int_{-\infty}^{0} \frac{\partial^{2} Z_{1}}{\partial y^{2}} d x+\left.b \mathscr{O} \frac{\partial Z_{1}}{\partial x}\right|_{x=0}
\end{gathered}
$$

The second order $y$-derivative term deserves special attention. This transverse gradient has large spatial dependence, attaining a wide range of values, from zero at the centerline (for symmetric axial forcing) to quite large values at the nozzle edges for large $P e$ values. That being noted, a transversely averaged form of Eq.(43) can be derived by integrating across the fuel and oxidizer port-domains, respectively, resulting in:

$$
\begin{gathered}
\left.\frac{1}{R_{f}} \int_{0}^{R_{f}} Z_{0}\right|_{x=0} d y-\int_{0}^{R_{f}}\left(\frac{1}{P e} \int_{-\infty}^{0} \frac{\partial^{2} Z_{0}}{\partial y^{2}} d x\right) d y-\int_{0}^{R_{f}}\left(\left.\frac{b}{P e} \frac{\partial Z_{0}}{\partial x}\right|_{x=0}\right) d y=Z_{f, r e s} \quad 0<|y|<R_{f} \\
\left.\frac{1}{R_{f}} \int_{R_{f}}^{R_{w}} Z_{0}\right|_{x=0} d y-\int_{R_{f}}^{R_{w}}\left(\frac{1}{P e} \int_{-\infty}^{0} \frac{\partial^{2} Z_{0}}{\partial y^{2}} d x\right) d y-\int_{R_{f}}^{R_{w}}\left(\left.\frac{b}{P e} \frac{\partial Z_{0}}{\partial x}\right|_{x=0}\right) d y=Z_{o x, r e s} \frac{\left(R_{w}-R_{f}\right)}{R_{f}} \quad R_{f}<|y|<R_{w}
\end{gathered}
$$

Defining the transversely averaged mixture fractions with a superscript " * ", and taking advantage of the no-flux boundary conditions at the centerline and solid port walls, these expressions become:

$$
\begin{array}{cc}
\left.\boldsymbol{Z}_{0}\right|_{x=0}{ }^{*}-\frac{b R_{f}}{P e}\left(\left.\frac{\partial Z_{0}}{\partial x}\right|_{x=0}\right)^{*}=Z_{f, r e s} & 0<|y|<R_{f} \\
\left.\boldsymbol{Z}_{0}\right|_{x=0}{ }^{*}-\frac{b R_{f}}{P e}\left(\left.\frac{\partial \mathcal{Z}_{0}}{\partial x}\right|_{x=0}\right)^{*}=Z_{o x, r e s} & R_{f}<|y|<R_{w}
\end{array}
$$

This expression is identical in form to the inflow boundary condition utilized by Tyagi et al. [26], whom prescribed the following condition locally:

$$
\begin{array}{ll}
\left.Z_{0}\right|_{x=0}-\left.\frac{R_{f}}{P e} \frac{\partial Z_{0}}{\partial x}\right|_{x=0}=Z_{f, r e s} & 0<|y|<R_{f} \\
\left.Z_{0}\right|_{x=0}-\left.\frac{R_{f}}{P e} \frac{\partial Z_{0}}{\partial x}\right|_{x=0}=Z_{o x, \text { res }} & R_{f}<|y|<R_{w}
\end{array}
$$


Although similar in form, these two sets of inflow boundary conditions are fundamentally different. Significantly, the latter set are local expressions, which physically state that at every transverse $y$ location, the instantaneous mass flux at the inlet plane, resulting from both convection and diffusion, matches the value at the reservoir. Comparing Eqs.(49) and (50) with Eq.(43) reveals that they are valid as integral expressions, but not locally. Transverse averaging allows us to eliminate the second derivative with respect to $y$ term by taking advantage of the no-flux boundary condition. Furthermore, analytically solving for the mixture fraction field solutions utilizing Eqs.(49) and (50) as the inlet boundary condition in place of Eq.(13), a process which is complicated by the fact that two additional solutions must be obtained in the port regions, results in discontinuous local mass flux values at $x=0$ for each $y$ station.

A series of analytic steady state mixture fraction field solutions can be obtained for the extended inlet geometry by replacing Eq.(13) with Eqs.(49) and (50) as an inlet boundary condition and solving Eq.(3) with axial diffusion included $(b=1)$. The new solution, valid for $x \geq 0 \& 0 \leq y \leq R_{w}$, can be obtained utilizing the same symmetry and no penetration boundary conditions as before. Additionally, fuel and oxidizer port solutions, valid for $x<0 \&$ $0 \leq y \leq R_{f}$ and $x<0 \& R_{f}<y \leq R_{w}$, respectively, can be obtained. Additional no penetration at the fuel port wall, $\partial Z_{0} /\left.\partial y\right|_{x<0, y=R_{f}}=0$, finite mixture fraction values, $\left.Z_{0}\right|_{x \rightarrow-\infty, y}=$ Finite, and mixture fraction matching,

$\left.Z_{0}^{f}\right|_{x=0,0 \leq y \leq R_{f}}=\left.\left.Z_{0}^{+}\right|_{x=0,0 \leq y \leq R_{f}} \& Z_{0}^{o x}\right|_{x=0, R_{f}<y \leq R_{w}}=\left.Z_{0}^{+}\right|_{x=0, R_{f}<y \leq R_{w}}$, boundary conditions must be applied to get meaningful solutions.

\section{Appendix B}

Presented are the details of the heat release transfer function low $S t$ asymptotics. Starting with an expansion of $z_{1}$ in the $P e \rightarrow \infty$, low St limit:

$$
Z_{1}(x, y, t)=-\varepsilon x \frac{\partial Z_{0}}{\partial x} \exp (-i \omega t)-\frac{i \pi \varepsilon S t_{R} x^{2}}{R_{f}} \frac{\partial Z_{0}}{\partial x} \exp (-i \omega t)+O\left(S t^{2}\right)
$$

Using Eq.(17) and the geometric relation, $\tan \theta_{0} \equiv d \xi_{0} / d x$, it can be shown that in the low St limit, the flame position fluctuation can be expressed in terms of the steady flame position as:

$$
\xi_{1, n}=-\left[1+\frac{i \pi S t_{R} x}{R_{f}}\right] \varepsilon \cdot \frac{d \xi_{0}}{d x} \cdot x \cdot \exp (-i \omega t)+O(S t)
$$

Noting that:

$$
\frac{\partial}{\partial x}\left[\frac{\partial Z_{0}\left(x, \xi_{0}(x)\right)}{\partial y}\right]=\frac{\partial^{2} Z_{0}\left(x, \xi_{0}(x)\right)}{\partial x \partial y}+\frac{d \xi_{0}}{d x} \frac{\partial^{2} Z_{0}\left(x, \xi_{0}(x)\right)}{\partial y^{2}}
$$

And utilizing these expressions, Eqn.(26) can be rewritten in the low St limit as:

$$
\frac{-\varphi_{o x}}{\left(1+\varphi_{o x}\right)^{2} \rho \partial s h_{R}} \dot{Q}_{1}(t)=-\int_{0}^{L_{f, 0} \pm} x \frac{\partial}{\partial x}\left(\frac{\partial Z_{0}\left(x, \xi_{0}(x)\right)}{\partial y}\right)\left[1+\frac{i \pi S t_{R} x}{R_{R}}\right] \varepsilon \exp (-i \omega t) d x
$$

This integral can be simplified using integration by parts, and results in: 


$$
\frac{-\varphi_{o x}}{\left(1+\varphi_{o x}\right)^{2} \rho \partial h_{R}} \dot{Q}_{1}(t)=\int_{0}^{L_{f, 0}{ }^{ \pm}} \frac{\partial Z_{0}\left(x, \xi_{0}(x)\right)}{\partial y} \varepsilon \exp (-i \omega t) d x+\int_{0}^{L_{f, 0 \pm}} \frac{2 \pi i S t_{R}}{R_{f}} x \frac{\partial Z_{0}\left(x, \xi_{0}(x)\right)}{\partial y} \varepsilon \exp (-i \omega t) d x
$$

Notice how the leading order term resembles the steady state heat release, $Q_{0}$. Thus putting this in terms of the transfer function:

$$
\mathscr{F}=1+i S t_{L f} \frac{2 \pi \int_{0}^{L_{f, 0}} x \frac{\partial Z_{0}\left(x, \xi_{0}(x)\right)}{\partial y} \cdot d x}{L_{f, 0} \int_{0}^{L_{f, 0}} \frac{\partial Z_{0}\left(x, \xi_{0}(x)\right)}{\partial y} \cdot d x}+O\left(S t_{L f}^{2}\right)
$$

\section{Appendix C}

This appendix presents the steady state mixture fraction solution for an unconfined system, that excludes axial diffusion, and utilizes the piecewise linear inlet boundary condition shown in Figure 14.

$$
Z_{0}\left(x^{*}, y^{*}\right)=\frac{1}{2}\left[\begin{array}{l}
{\left[\frac{y^{*}+1+\delta}{2 \delta}\right] \operatorname{erf}\left(\frac{\left(-1+\delta-y^{*}\right)}{2 \sqrt{x^{*}}}\right)-\frac{x^{*}}{\delta} \exp \left(-\frac{\left(y^{*}+1+\delta\right)^{2}}{4 x^{*}}\right)+} \\
-\left[\frac{y^{*}+1+\delta}{2 \delta}\right] \operatorname{erf}\left(\frac{\left(-1-\delta-y^{*}\right)}{2 \sqrt{x^{*}}}\right)+\frac{x^{*}}{\delta} \exp \left(-\frac{\left(y^{*}+1-\delta\right)^{2}}{4 x^{*}}\right)+ \\
+\left[\frac{-y^{*}+1+\delta}{2 \delta}\right] \operatorname{erf}\left(\frac{\left(1+\delta-y^{*}\right)}{2 \sqrt{x^{*}}}\right)+\frac{x^{*}}{\delta} \exp \left(-\frac{\left(y^{*}-1+\delta\right)^{2}}{4 x^{*}}\right)+ \\
-\left[\frac{-y^{*}+1+\delta}{2 \delta}\right] \operatorname{erf}\left(\frac{\left(1-\delta-y^{*}\right)}{2 \sqrt{x^{*}}}\right)-\frac{x^{*}}{\delta} \exp \left(-\frac{\left(y^{*}-1-\delta\right)^{2}}{4 x^{*}}\right)
\end{array}\right]
$$

where non-dimensional coordinates are being utilized for compactness and are defined as follows:

$$
x^{*}=\frac{x}{P e R_{f}} \quad y^{*}=\frac{y}{R_{f}}
$$




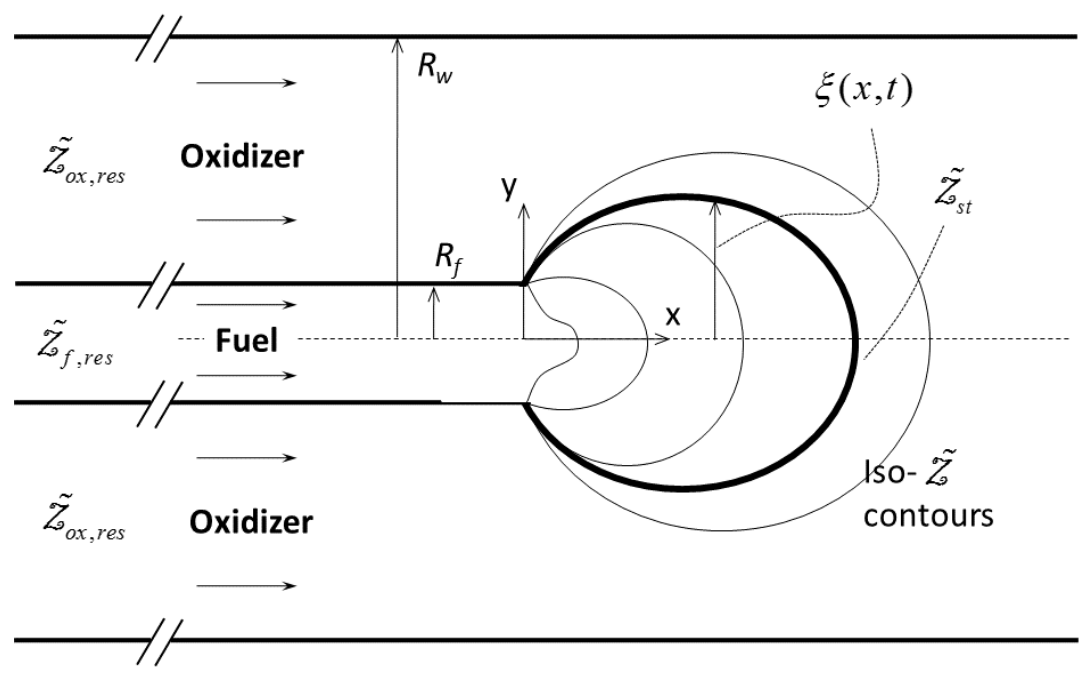

Figure 1. Schematic of the non-premixed flame extended inlet model problem utilized to capture axial diffusion effects. Fuel and oxidizer reservoirs and ports, $x<0$, have been added to our previously considered domain.

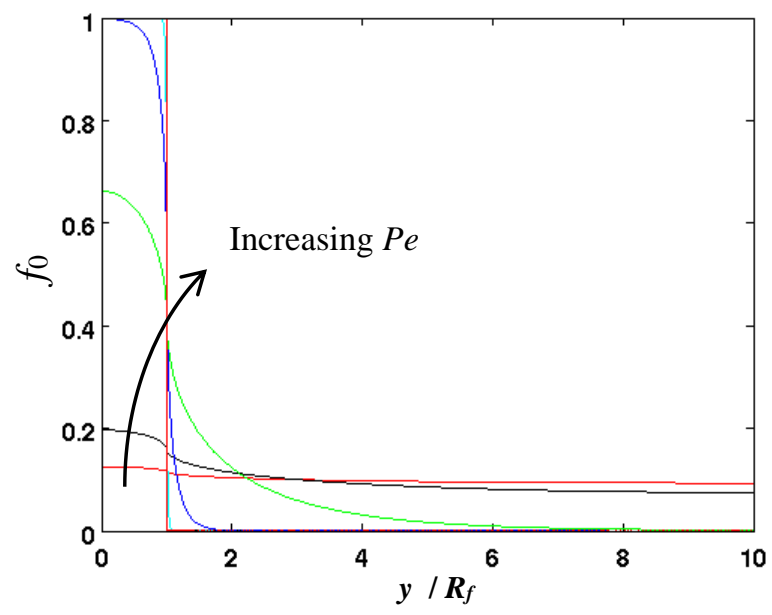

Figure 2. Computationally extracted steady state inlet mixture fraction profiles, $f_{0}(y)$, for various degrees of axial diffusion, for an extended inlet geometry defined by $R_{w} / R_{f}=10$. Curves are shown for $P e$ values ranging from $10^{-2}$ to $10^{4}$ in powers of 10 . 


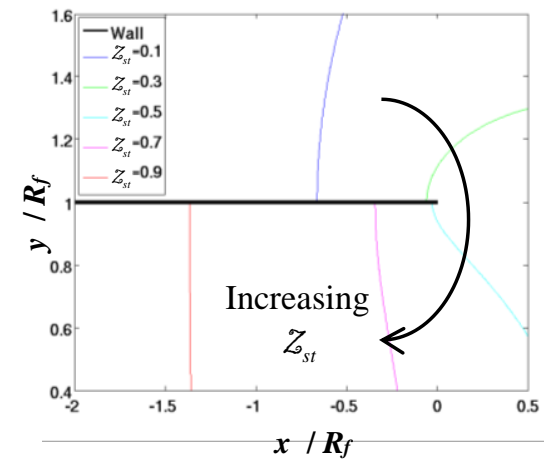

Figure 3. Unforced flame attachment point dependence upon $Z_{s t}$ for $P e$ value of 1 . The curves shown are for $Z_{s t}$ values of $0.1,0.3,0.5,0.7$, and 0.9 respectively, where the arrow indicates the direction of increasing $Z_{s t}$.

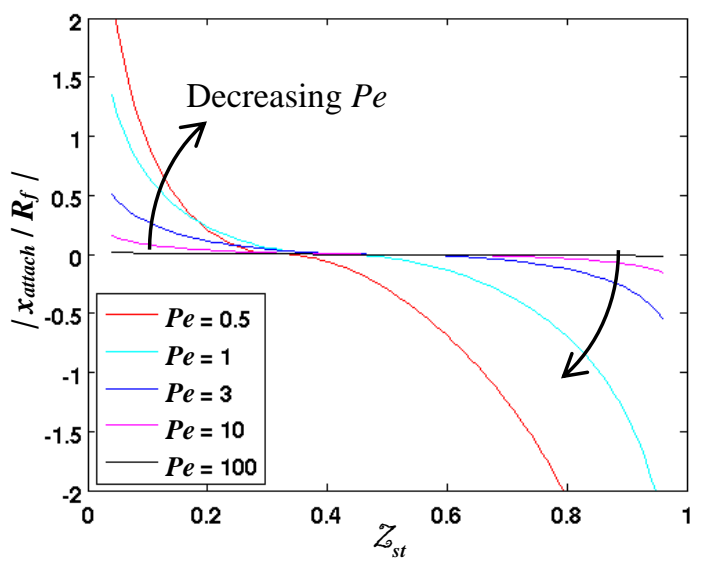

Figure 4. Steady state flame attachment point summary plot of $x_{\text {attach }} / R_{f}$ (location where $x<0$ and $y=R_{f}$ ) versus $Z_{s t}$ for several different $P e$ values. The curves shown are for $P e$ values of $0.5,1,3,10$, and 100 respectively, where the arrows indicate the direction of decreasing $P e$. Positive and negative values represent attachment locations inside the oxidizer port on the top side of the fuel port lip, and inside the fuel port on the bottom side of the fuel port lip, respectively.
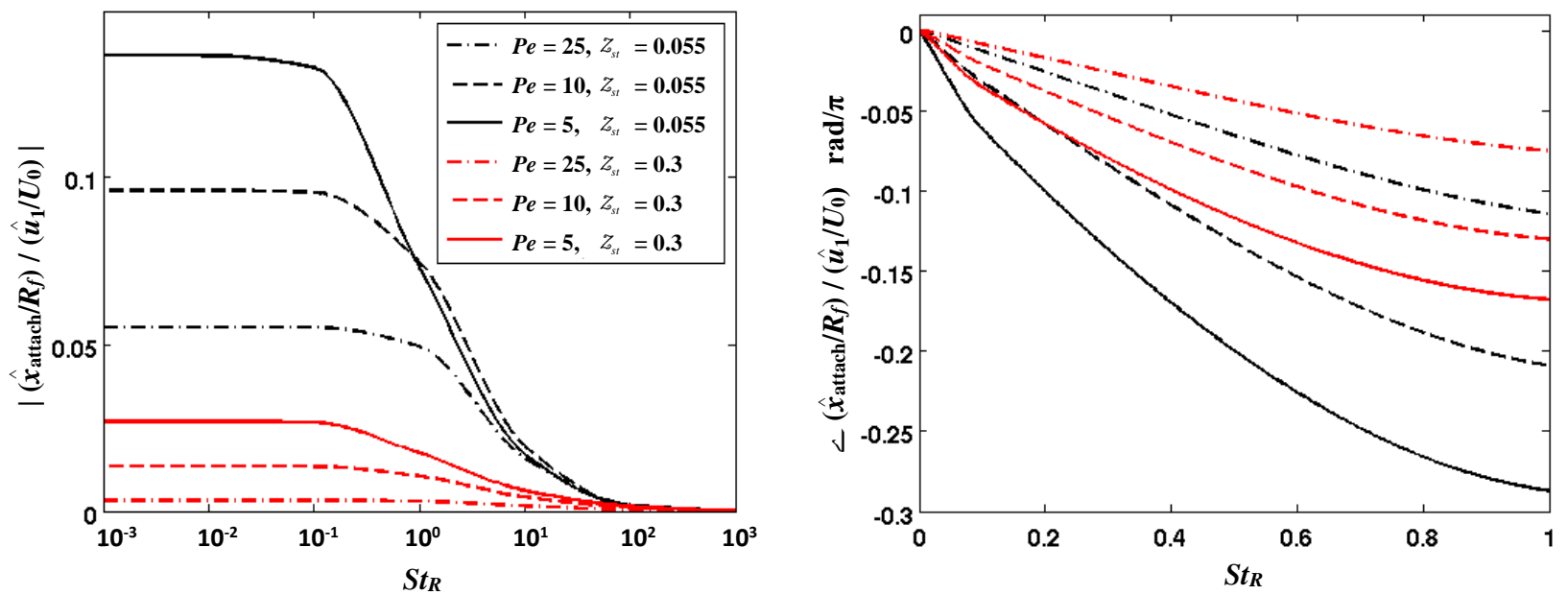

Figure 5. Magnitude and phase of the instantaneous attachment point (i.e., flame base position) transfer function. Line color and style distinguish between the various curves $Z_{s t}$ and $P e$ values, respectively. 

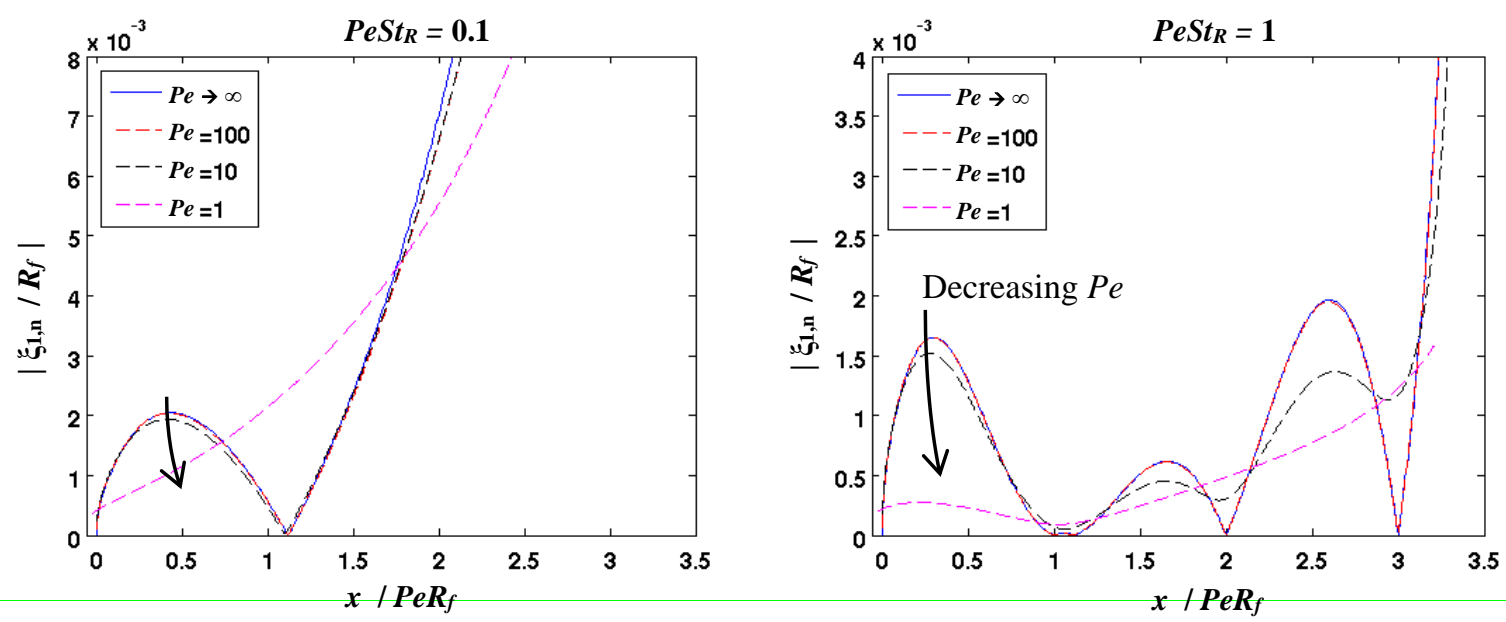

Figure 6. Axial dependence of the flame response magnitude for a representative over-ventilated flame, $Z_{s t}=\mathbf{0 . 3}$, for various degrees of axial diffusion. Arrows indicate direction of decreasing $P e$ value.
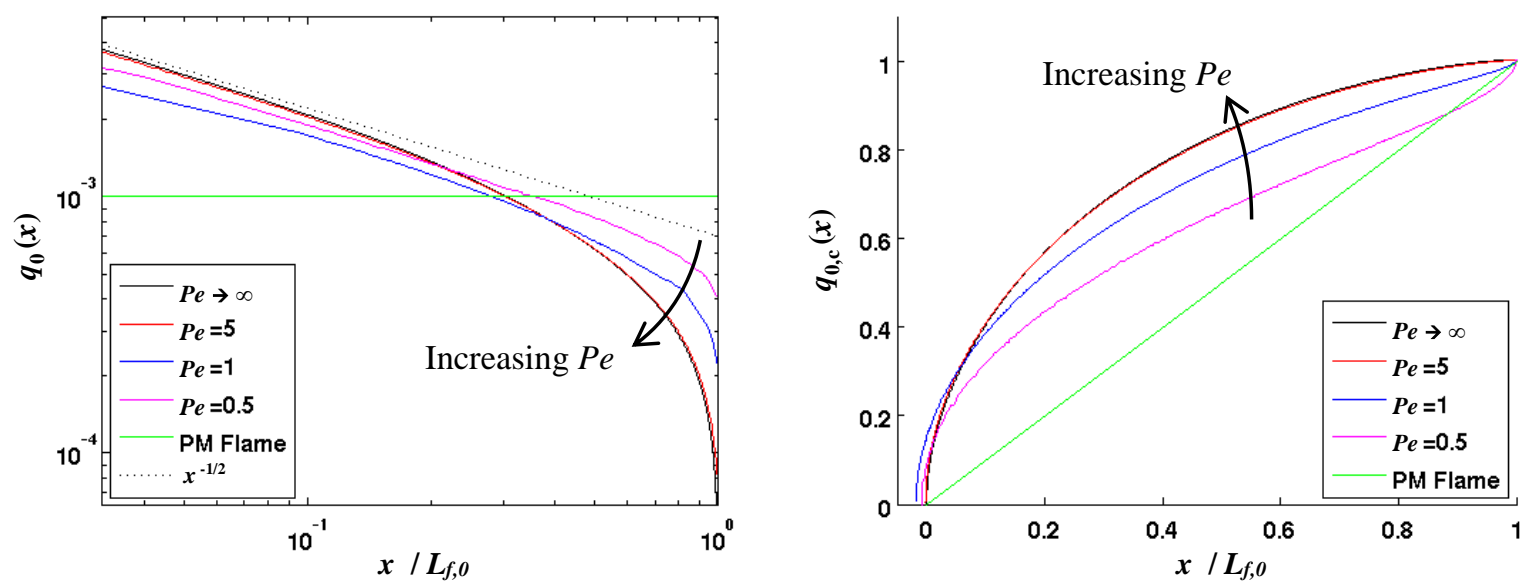

Figure 7. Axial dependence of steady state heat release, both distributed and cumulative, for $Z_{s t}=0.3$ and various $P e$ values. The numerical $P e$ value curves $(0.5,1,5)$ are computational results, while the $P e \rightarrow \infty$ curve is an analytical result. 

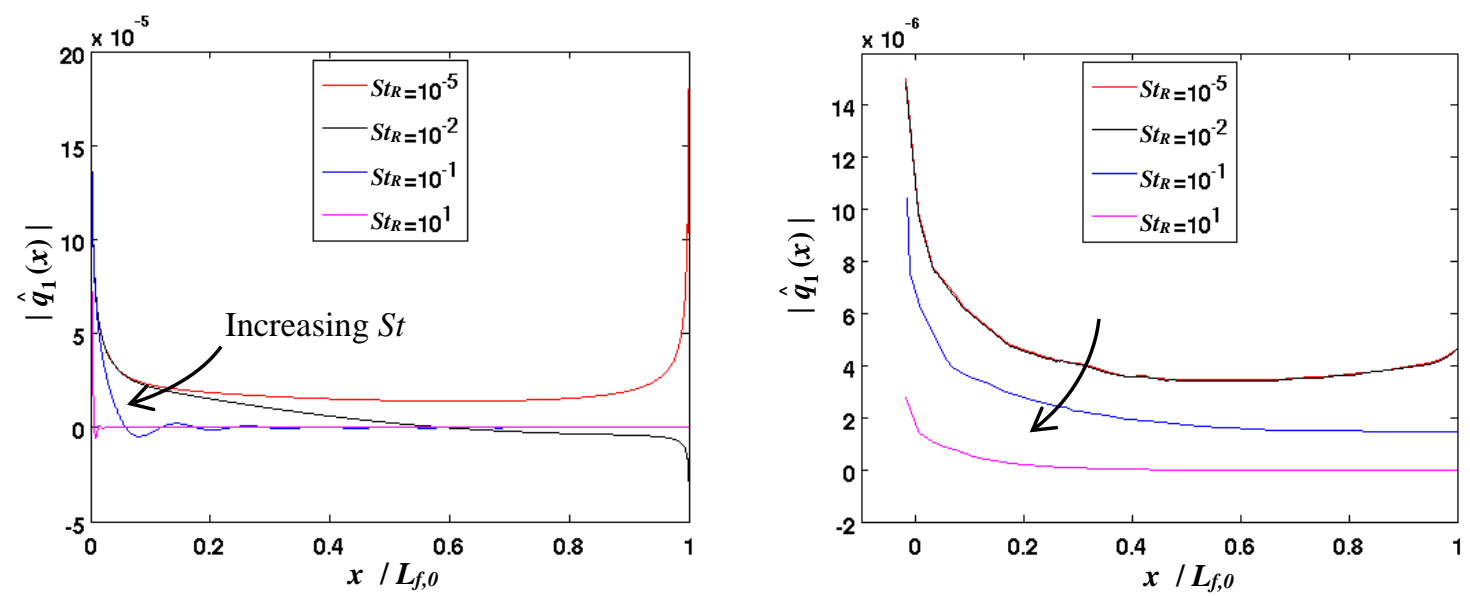

Figure 8. Axial dependence of fluctuating heat release distributions for $P e$ values of 25 (left) and 1 (right) and various values $S t$. Arrows indicate direction of increasing $S t$ value. Similar curves for the $P e \rightarrow \infty$ analytical results were omitted due to practical coincidence with the $P e=25$ results.
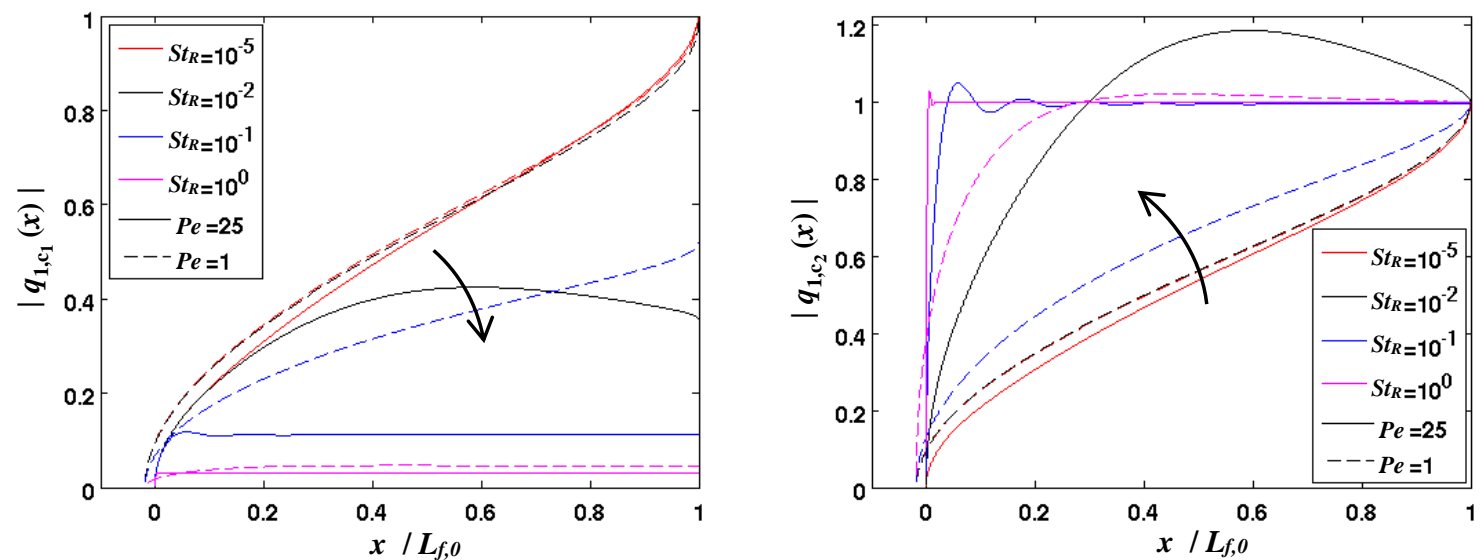

Figure 9. Axial dependence of fluctuating cumulative heat release distributions normalized by $q_{1, c_{1}}($ left) and $q_{1, c_{2}}$ (right) for various values of $P e$ and $S t$. Arrows indicate direction of increasing $S t$ value. Similar curves for the $P e \rightarrow \infty$ analytical results were omitted due to practical coincidence with the $P e=25$ results.
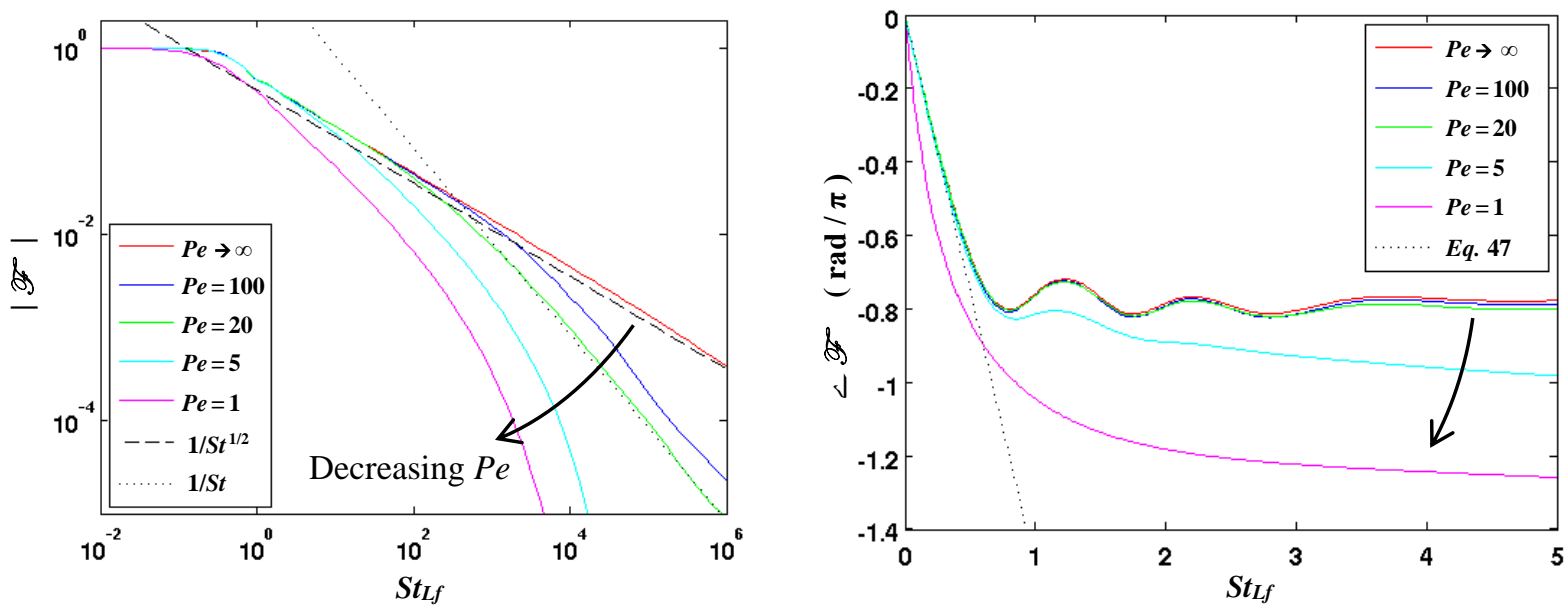

Figure 10. Heat release transfer function amplitude (left) and phase (right) as a function of $S t_{L_{f}}$ for $Z_{s t}=0.3$ for various $P e$ values. Arrows indicate direction of decreasing $P e$ value. 


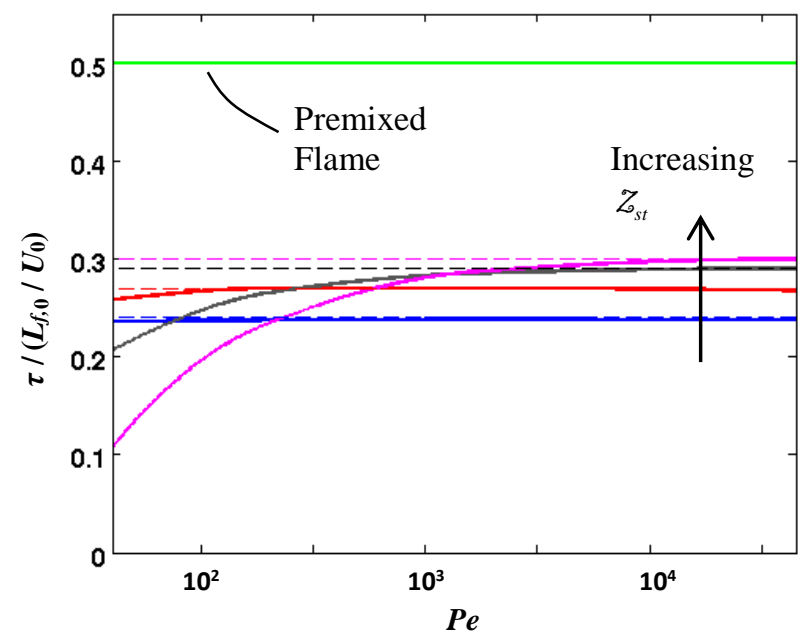

Figure 11. Normalized time delay parameter, $\tau /\left(L_{f, 0} / U_{0}\right)$, shown as a function of $\boldsymbol{P e}$ for various $Z_{s t}$ values $(0.3,0.6,0.8,0.9)$. Arrow indicates direction of increasing $Z_{s t}$ value. The corresponding (based on geometry, assumptions, and configuration) premixed flame value is also displayed. For each $Z_{s t}$ value, the corresponding $P e \rightarrow \infty$ analytical solution is shown by a dashed line.

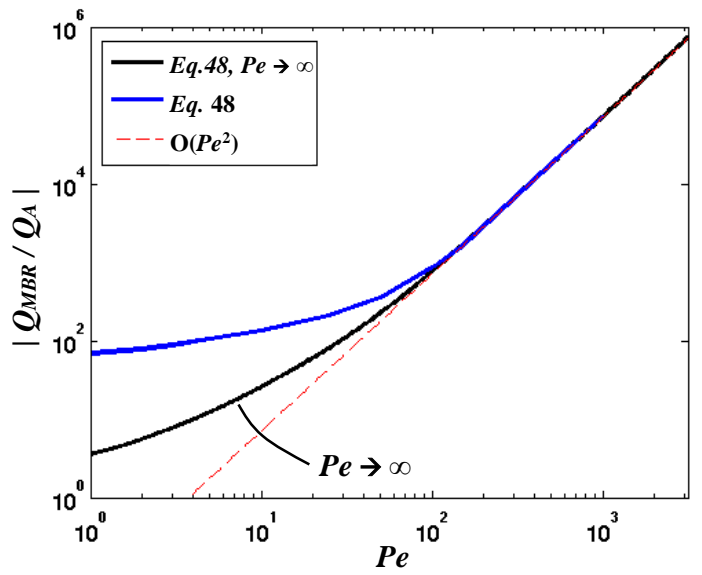

Figure 12. Ratio of mass burning rate to area terms in the unsteady heat release expression for the computed extended inlet and $P e \rightarrow \infty$ limiting solution cases. 


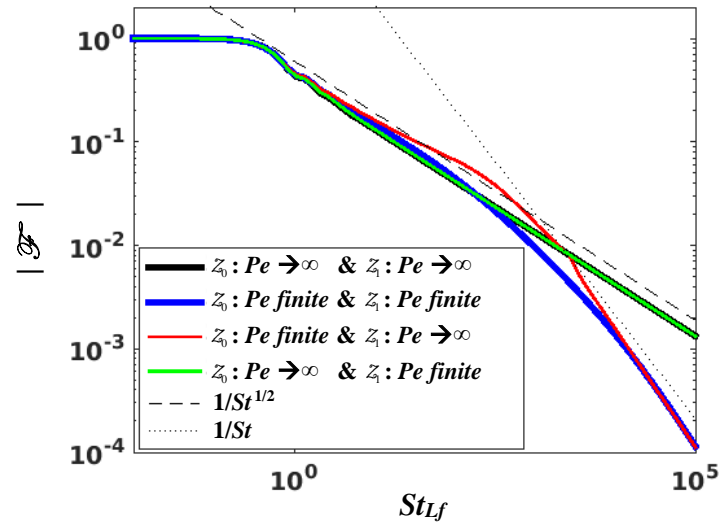

Figure 13. Flame transfer function amplitude curves isolating dynamical and inflow boundary effects on high St asymptotic trends (see text for description of different curves).

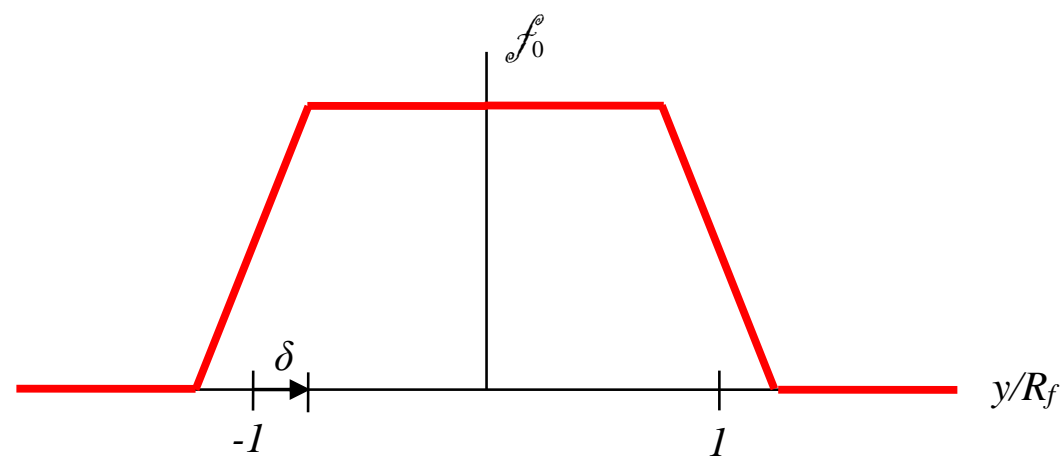

Figure 14. Piecewise linear inlet mixture fraction boundary condition utilized to analytically evaluate the effects of a smoothed inflow profile on high $S t$ asymptotic trends.

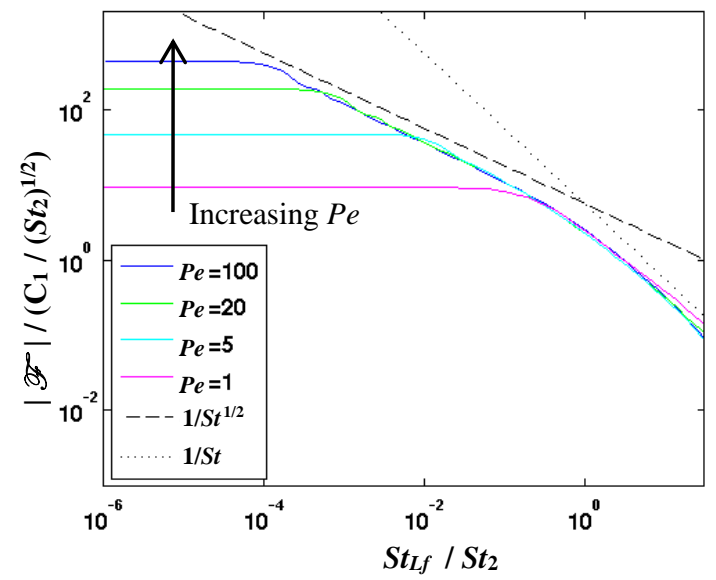

Figure 15. Normalized heat release transfer function amplitude plot for various degrees of axial diffusion, i.e. $P e$ values, using the high Strouhal number scalings for normalization. The $1 / S t^{1 / 2}$ and $1 / S t$ lines are the asymptotic values for the $\boldsymbol{P e}=\mathbf{2 0}$ case for visual representation. Arrow indicates direction of increasing $P e$ value. 